4-1-1999

\title{
On the Detachment of Step-Tapered Doublers : Part 1-Foundations
}

\author{
W. J. Bottega \\ Rutgers University - New Brunswick/Piscataway \\ Anette M. Karlsson \\ Cleveland State University, a.karlsson@csuohio.edu
}

Follow this and additional works at: https://engagedscholarship.csuohio.edu/enme_facpub

Part of the Mechanical Engineering Commons

How does access to this work benefit you? Let us know!

\section{Publisher's Statement}

NOTICE: this is the author's version of a work that was accepted for publication in International Journal of Solids and Structures. Changes resulting from the publishing process, such as peer review, editing, corrections, structural formatting, and other quality control mechanisms may not be reflected in this document. Changes may have been made to this work since it was submitted for publication. A definitive version was subsequently published in International Journal of Solids and Structures, 36, 11, (04-01-1999); 10.1016/S0020-7683(98)00052-3

\section{Original Citation}

Bottega, W. J., and Karlsson, A. M., 1999, "On the Detachment of Step-Tapered Doublers : Part 1-foundations," International Journal of Solids and Structures, 36(11) pp. 1597-1623.

This Article is brought to you for free and open access by the Mechanical Engineering Department at EngagedScholarship@CSU. It has been accepted for inclusion in Mechanical Engineering Faculty Publications by an authorized administrator of EngagedScholarship@CSU. For more information, please contact library.es@csuohio.edu. 


\title{
On the detachment of step-tapered doublers: Part 1-foundations
}

\author{
W. J. Bottega*, A. M. Karlsson \\ Department of Mechanical and Aerospace Engineering, Rutgers University, Piscataway NJ 08854-8058, U.S.A.
}

\section{Introduction}

The configuration of a secondary structure, a 'doubler', adhered to a primary structure occurs in many settings. A particular contemporary system of this type, which is of current interest, is that corresponding to a composite 'patch' adhered to a damaged (cracked) structure in an attempt to transfer the load from the base structure and alleviate the stress intensity in the vicinity of the

*Corresponding author. Fax: 001732445 5313; e-mail : bottega@rci.rutgers.edu 
damage. Typically the patch is designed and its effectiveness evaluated toward this end by considering plane stress type analyses of flat structures subjected to in-plane loading. We cite here the work of Roderick (1980), Sih and Hong (1989), and Baker (1993), as examples. A discussion of these and other related papers is presented in the papers by Bottega (1995), and Bottega and Loia $(1996,1997)$, and hence is not elaborated upon presently.

Debonding of a patch from its base structure is evidently a pertinent issue as such behavior can have dramatic consequences with regard to the effectiveness of the doubler and the integrity of the overall structure. The issue of edge debonding of untapered patches from base structures has been considered for flat structures by Bottega (1995) and Bottega and Loia (1997), and for cylindrical structures by Bottega and Loia (1996). Caveats regarding extrapolation from simplified representations/tests were presented by Loia and Bottega (1995). In all cases, various loading and support conditions were considered and it was seen that out of plane bending together with inplane stretching strongly influence the debonding behavior of the evolving structure. A variety of characteristics of the debonding structures were exhibited in these studies including the existence and influence of a contact zone under appropriate conditions, the phenomenon of edge contact, the influence of support conditions, and the influence of the relative patch stiffness and length on the evolution of the composite structure. With regard to the latter effects, it was seen that relatively stiff patches generally debond more readily, and in a more unstable fashion, than their more compliant counterparts. In anticipation of undesirable behavior due to the presence of stress concentrations at discontinuous geometries, tapering of the edge of the patch to reduce this effect is currently being explored in certain tests and designs [e.g., (Raizenne et al., 1995)]. We note that a number of studies of tapered laminated structures, usually of flat symmetric lay-up configurations subjected to in-plane tension-compression loading, have been conducted for structural elements where a change in cross section is imposed as a result of functional requirements (see, for example, Fish and Lee, 1989; Armanios and Pernas, 1989; Salpekar et al., 1991 ; Curry et al., 1992 ; Botting et al., 1996). Consequently, it is of interest to understand how such tapering of the edge of the doubler will alter the debonding behavior of patched structures. We therefore consider such issues in the present study.

Specifically, we consider both flat and cylindrical structures (see Fig. 1) to which multilayer patches are adhered, for the situation where the edges of the patches are tapered layerwise (see Fig. 2). The formulation for both flat and curved structures will be considered simultaneously. Three loading types will be considered. These include (i) applied in-plane/circumferential tension, (ii) three-point transverse loading, and (iii) applied transverse (internal) pressure. As in the related studies pertaining to uniform untapered patches (Bottega, 1995; Bottega and Loia, 1996; Bottega and Loia, 1997) the problem is formulated from a unified point of view, as a moving intermediate boundaries problem in the calculus of variations - with an appropriate thin structure theory used to model the base structure and each of the layers of the patch individually, and a Griffith type energy criterion incorporated to govern debonding of the patch from the base structure. The existence and effects of the presence of a contact zone is included as are the phenomena of edge point contact and lift. Such an approach yields a self-consistent formulation for the evolving composite structure which includes the governing equations and boundary and matching conditions for the intact segment of the composite structure, for the debonded segment of the composite structure in a region of sliding contact, and for the base structure and composite patch in the region of separation. It also yields the transversality conditions which define the locations of 


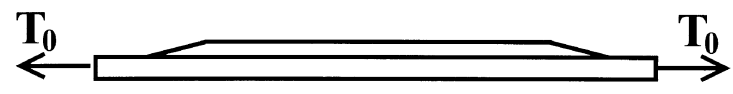

(a)

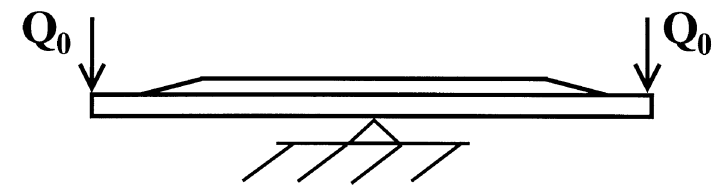

(b)

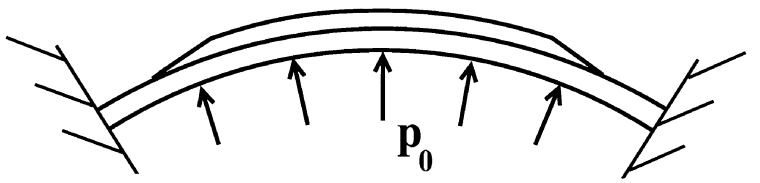

(c)

Fig. 1. Structures with edge tapered patches subjected to various loading conditions. (a) Applied in-plane tension (shown for flat configuration), (b) three-point transverse loading (shown for flat configuration), (c) applied (internal) pressure (shown for cylindrical structure with clamped-fixed supports).

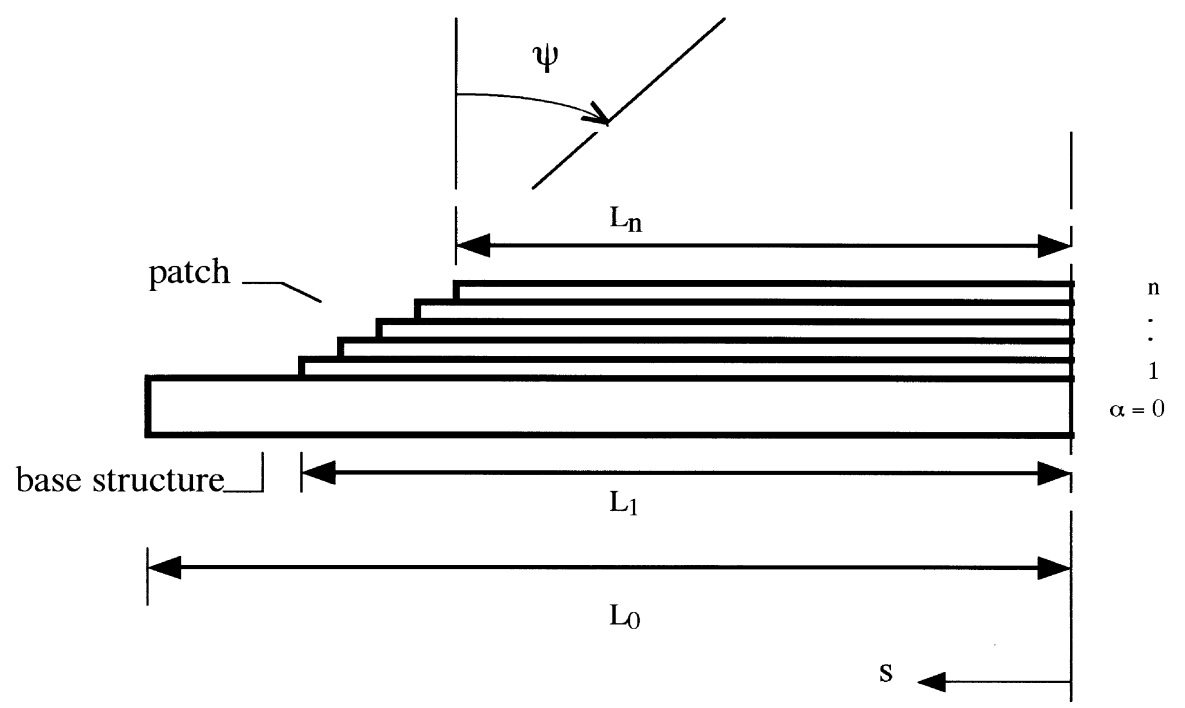

Fig. 2. Geometry for step tapered patch with base panel. (Shown for flat panel but identical for cylindrical panel.)

the propagating lift zone/contact zone boundary and the propagating contact zone/bond zone boundary, corresponding to equilibrium configurations of the evolving composite structure. The condition for the latter yields the self-consistent and physically interpretable expressions for the corresponding energy release rates for debonding. Finally, results of numerical simulations for the 
test configurations corresponding to patched plates subjected to applied in-plane tensile loading and three-point transverse loading are presented for various taper angles and compared. The complex results of intricate simulations pertaining to patched plates and shells subjected to applied transverse pressure are presented in Part 2 of this study (Karlsson and Bottega, 1998). In all, the influence of support conditions is included. The studies reveal interesting, and often unanticipated, characteristics associated with the degree of taper, particularly for the case of pressure loading.

\section{Formulation}

We next present a unified formulation for the class of structures of interest. Both flat and curved structures will be considered simultaneously, though the length normalization will differ for the two structural types in keeping with prior related work (Bottega, 1995; Bottega and Loia, 1996; Bottega and Loia, 1997; and Loia and Bottega, 1995).

Consider the thin cylindrical (flat) structure comprised of a base shell (plate) of normalized half-span $L_{0}$ to which a cylindrical (flat) patch of half-span $L_{1} \leqslant L_{0}$ is adhered. Let the patch be comprised of $n$ layers, numbered $\alpha=1,2, \ldots, n$ proceeding away from the base structure in the transverse direction, and let the corresponding lengths $L_{\alpha}$ be sequentially shorter such that $L_{1}>L_{2}>\cdots>L_{n}$, as shown in Fig. 2. Let the patch be bonded to the base structure over the region $S_{1}: s \in[0, a]$ as shown in Fig. 3, where $s$ is the circumferential arc (axial) coordinate measured from centerspan of the structure. Further, let us consider the debonded/unbonded portion of the patch to maintain sliding contact over the region $S_{2}: s \in[a, b]$ immediately ahead of the bonded region, while the portion of the patch defined on $S_{3}: s \in\left[b, L_{0}\right]$ is lifted/separated from the base structure. These three regions will be referred to as the 'bond zone', 'contact zone' and 'lift zone', respectively. We designate the domain of definition of portion of the patch in the lift zone as $S_{31}: s \in\left[b, L_{1}\right]$ such that $S_{31} \subset S_{3}$. However, when referring to the portion of the patch in region $S_{3}$, it will be understood that the corresponding subregion is indicated. At this point, let us also define the 'conjugate bond zone size' $a^{*}=L_{0}-a$ as indicated in the figure. We shall be interested in examining the evolution and response of the 'composite structure' when it is subjected to (i) applied circumferential/in-plane tension, (ii) three-point transverse loading, and (iii) applied transverse (internal) pressure. In what follows all length scales are normalized with respect to the dimensional radius $\bar{R}$ of the undeformed shell segment (or by the dimensional half length $\overline{L_{0}}$ for the flat structure), and the common surface or interface between the patch and base structure, and its extension, will be used as the reference surface.

The individual patch layers as well as the base structure will be modeled as von Karman plates for the case of a flat structure (Bottega, 1995) or by the shallow shell model employed in Bottega and Loia (1996) for the case of cylindrical panels. The corresponding relations for the normalized (centerline) membrane strains $e_{0 i}(s)$ and $e_{\alpha i}(s)$ and the normalized curvature changes $\kappa_{0 i}(s)$ and $\kappa_{\alpha i}(s)$ for the base structure and layer $\alpha$ of the patch in each region are thus, respectively, given by

$$
\begin{aligned}
& e_{0 i}=u_{0 i}^{\prime}-k w_{0 i}+\frac{1}{2} w_{0 i}^{\prime 2}, \quad \kappa_{0 i}=w_{0 i}^{\prime \prime}+k w_{0 i}, \quad s \in S_{i} \quad(i=1-3) \\
& e_{\alpha i}=u_{\alpha i}^{\prime}-k w_{\alpha i}+\frac{1}{2} w_{\alpha i}^{\prime 2}, \quad \kappa_{\alpha i}=w_{\alpha i}^{\prime \prime}+k w_{\alpha i}, \quad s \in S_{i \alpha} \quad(i=1-3, \alpha=1,2, \ldots, n)
\end{aligned}
$$




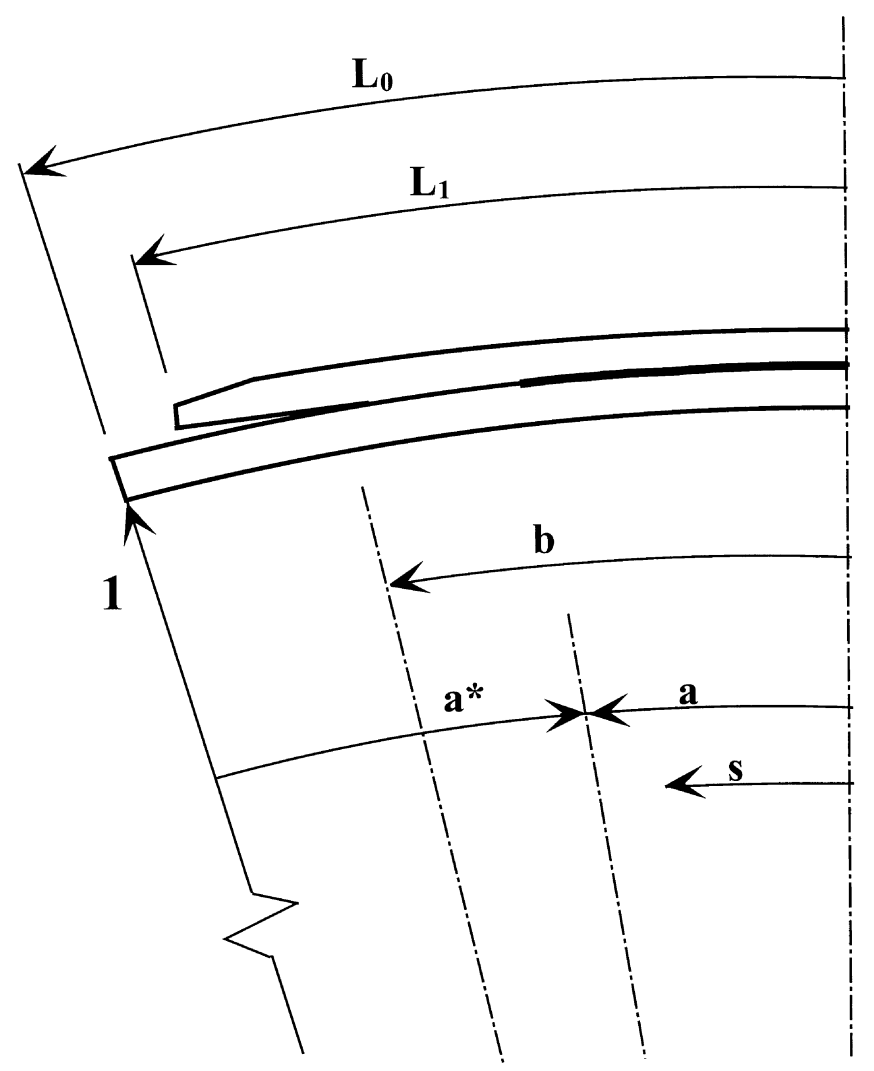

Fig. 3. Half-span of structure showing characteristic lengths and coordinates. (Shown for curved panel.)

where $k=0$ or 1 , with $k=0$ corresponding to the case of a flat structure and $k=1$ to the case of a cylindrical structure.

In eqns (1a)-(1d), $u_{0 i}=u_{0 i}(s)$ (positive in direction of increasing $s$ ) and $w_{0 i}=w_{0 i}(s)$ (positive inward/downward), respectively, correspond to the circumferential (in-plane) and transverse displacements of the centerline of the base panel in region $S_{i}$, and $u_{\alpha i}=u_{\alpha i}(s)$ and $w_{\alpha i}=w_{\alpha i}(s)$ correspond to the analogous displacements of the centerline of layer $\alpha$ of the patch. In addition, superposed primes indicate total differentiation with respect to $s$.

In keeping with the Kirchoff Hypothesis, the circumferential (axial) displacements and membrane strains, $u_{i}(r, s)$ and $e_{i}(r, s)$, are taken to vary linearly through the thickness of each layer of the patch and base structure while the through the thickness dependence of the transverse displacements $w_{i}(r, s)$ is considered to be of higher order. Hence,

$$
\begin{aligned}
& u_{i}=u_{\alpha i}+z_{\alpha} w_{\alpha i}^{\prime} ; \quad-\frac{h_{\alpha}}{2} \leqslant z_{\alpha} \leqslant \frac{h_{\alpha}}{2}, \quad(i=1-3, \alpha=0,1, \ldots, n) ; \\
& e_{i}=e_{\alpha i}+z_{\alpha} \kappa_{\alpha i} ; \quad-\frac{h_{\alpha}}{2} \leqslant z_{\alpha} \leqslant \frac{h_{\alpha}}{2}, \quad(i=1-3, \alpha=0,1, \ldots, n) ;
\end{aligned}
$$


where $z_{\alpha}=z-\rho_{\alpha}=(r-k)-\rho_{\alpha}$. The parameter $\rho_{\alpha}$ radially (transversally) locates the centroid of layer $\alpha(\alpha=0,1,2, \ldots, n)$ of the structure with respect to the reference surface, while $z_{\alpha}$ is the transverse coordinate measured from the centroid of layer $\alpha, z$ is the corresponding transverse coordinate measured from the reference surface, and $r$ is the transverse coordinate measured from the origin (the center of curvature of the undeformed cylinder, or directly from the reference surface for the flat plate). The index $\alpha=0$ corresponds to the base structure.

It follows that the displacements $u_{0 i}(s)$ and the membrane strains $e_{0 i}(s)$ at the base structure centerline are related to their counterparts at the reference surface, $u_{i}^{*}(s)$ and $e_{i}^{*}(s)$, by the relations

$$
u_{i}^{*}=u_{0 i}+\frac{h_{0}}{2} w_{0 i}^{\prime}, \quad e_{i}^{*}=e_{0 i}+\frac{h_{0}}{2} \kappa_{0 i}, \quad(i=1-3) .
$$

Further, the parameters $h_{0} \ll 1$ and $h_{\alpha} \ll 1(\alpha=1,2, \ldots, n)$ correspond to the normalized thicknesses of the base panel and of layer $\alpha$ of the patch, respectively.

The normalized resultant membrane forces in the base panel and layer $\alpha$ of the patch in region $S_{i}(i=1-3)$ are then given, respectively, as

$$
N_{0 i}(s)=C_{0} e_{0 i}(s) \quad \text { and } \quad N_{\alpha i}(s)=C_{\alpha} e_{\alpha i}(s) \quad(i=1-3, \alpha=1,2, \ldots, n)
$$

where $C_{0}$ and $D_{0}$, respectively, represent the normalized membrane stiffness and bending stiffness of the base panel, and $C_{\alpha}$ and $D_{\alpha}$, represent the corresponding normalized membrane and bending stiffnesses of layer $\alpha$ of the patch $(\alpha=1,2, \ldots, n)$. In what follows, the normalization of the stiffnesses of the primitive structures is based on the dimensional bending stiffness $\bar{D}_{0}$ of the base panel, and the dimensional radius $\bar{R}$ for the cylindrical structure (or the half length $\bar{L}_{0}$ for the flat structure) in the undeformed configuration. Hence,

$$
C_{0}=12 / h_{0}^{2}, \quad D_{0}=1, \quad C_{\alpha}=C_{0} E_{\alpha} t_{\alpha}, \quad D_{\alpha}=E_{\alpha} t_{\alpha}^{3}, \quad(\alpha=1,2, \ldots, n)
$$

where:

$$
t_{\alpha}=h_{\alpha} / h_{0}, \quad E_{\alpha}=\bar{E}_{\alpha} / \bar{E}_{0} \quad \text { or } E_{\alpha}=\frac{\bar{E}_{\alpha} /\left(1-v_{\alpha}^{2}\right)}{\bar{E}_{0} /\left(1-v_{0}^{2}\right)}, \quad(\alpha=1,2, \ldots, n)
$$

$\bar{E}_{0}$ and $\bar{E}_{\alpha}$ correspond to the (dimensional) elastic moduli of the base panel and layer $\alpha$ of the patch, respectively, and $v_{0}$ and $v_{\alpha}$ correspond to the associated Poisson's ratios.

We next formulate an energy functional $\Pi$, as follows :

$$
\Pi=\sum_{i=1}^{3}\left\{U_{0 B}^{(i)}+U_{0 M}^{(i)}\right\}+\sum_{i=1}^{3} \sum_{\alpha=1}^{q \leqslant n}\left\{U_{\alpha,}^{(i)}+U_{\alpha M}^{(i)}-\Lambda_{\alpha}^{(i)}\right\}-\mathscr{W}+\Gamma
$$

where $n$ is the maximum number of layers of the patch, $q=q(s) \leqslant n$ is the maximum (outermost) layer number at a particular circumferential (axial) coordinate ' $s$ '. Further,

$$
U_{0 B}^{(i)}=\int_{S_{i}} \frac{1}{2} D_{0} \kappa_{0 i}^{2} \mathrm{~d} s, \quad U_{\alpha B}^{(i)}=\int_{S_{i}} \frac{1}{2} D_{\alpha} \kappa_{\alpha i}^{2} \mathrm{~d} s, \quad(i=1-3, \alpha=1,2, \ldots, n),
$$

respectively, correspond to the bending energies of the base panel, and of patch layer $\alpha$, in region $i$ 


$$
U_{0 M}^{(i)}=\int_{S_{i}} \frac{1}{2} C_{0} e_{0 i}^{2} \mathrm{~d} s, \quad U_{\alpha M}^{(i)}=\int_{S_{i}} \frac{1}{2} C_{\alpha} e_{\alpha i}^{2} \mathrm{~d} s, \quad(i=1-3, \alpha=1,2, \ldots, n)
$$

are the corresponding membrane energies of the base panel and patch layer $\alpha$, respectively, and $\Lambda_{\alpha}^{(i)}$ are constraint functionals, given by

$$
\Lambda_{\alpha}^{(i)}=\int_{S_{i}} \sigma_{\alpha}^{(i)}\left(w_{\alpha i}-w_{(\alpha-1) i}\right) \mathrm{d} s+\int_{S_{i}} \tau_{\alpha}^{(i)}\left(u_{\alpha i}-u_{(\alpha-1) i}\right) \mathrm{d} s, \quad(\alpha=1,2, \ldots, n)
$$

where $\sigma_{\alpha}^{(i)}$ and $\tau_{\alpha}^{(i)}$ are Lagrange multipliers such that $\tau_{1}^{(2)}=\tau_{1}^{(3)}=\sigma_{1}^{(3)}=0$ and $\sigma_{1}^{(2)}<0$. The Lagrange multipliers may be seen to correspond to the interface stresses. The functional $\mathscr{W}$ corresponds to the work done by the applied loading and is given by

$$
\mathscr{W}=T_{0} u_{03}\left(L_{0}\right), \quad \text { or } \quad \mathscr{W}=Q_{0} w_{03}\left(L_{0}\right), \quad \text { or } \quad \mathscr{W}=-\sum_{i=1}^{3}\left\{\int_{S_{i}} p_{0} w_{0 i} \mathrm{~d} s\right\}
$$

depending upon the specific problem of interest, where $T_{0}$ represents the normalized applied circumferential (in-plane) tension per unit width, $Q_{0}$ is the normalized intensity of the applied transverse loads for the 3-point case, and $p_{0}$ corresponds to the normalized applied (internal) pressure (see Fig. 1). Further,

$$
\Gamma=2 \gamma\left(a^{*}-a_{0}^{*}\right)
$$

is the delamination energy, where

$$
a^{*}=L_{0}-a
$$

is the 'conjugate' bond zone (half) length as defined earlier, $a_{0}^{*}$ corresponds to some initial value of $a^{*}$, and $\gamma$ is the normalized bond energy (bond strength) ${ }^{1}$ associated with the patch-base panel interface. $^{2}$

The normalized loads $T_{0}, Q_{0}$ and $p_{0}$ appearing in eqns $(9 \mathrm{a}, \mathrm{b}, \mathrm{c})$ are related to their dimensional counterparts $\bar{T}, \bar{Q}$ and $\bar{p}$, by

$$
T_{0}=\bar{T} \bar{R}^{2} / \bar{D}_{0}, \quad Q_{0}=\bar{Q} \bar{R}^{2} / \bar{D}_{0}, \quad p_{0}=\bar{p} \bar{R}^{3} / \bar{D}_{0},
$$

where, we recall that, $\overline{D_{0}}$ is the dimensional bending stiffness of the base panel. In a similar manner, the normalized bond energy $\gamma$ is related to its dimensional counterpart $\bar{\gamma}$, by

$$
\gamma=\bar{\gamma} \bar{R}^{2} / \bar{D}_{0}
$$

The normalized interfacial stresses $\sigma_{\alpha}^{(i)}$ and $\tau_{\alpha}^{(i)}(i=1-3, \alpha=1,2, \ldots, n)$, i.e.- the Lagrange multipliers, are related to their dimensional counterparts in a manner analogous to that of the applied

\footnotetext{
${ }^{1}$ More generally, the bond strength $\gamma$ may be considered to be an implicit function of $a^{*}$ such that $\Gamma$ is defined in terms of its variation, viz $\delta \Gamma=2 \gamma \delta a^{*}$, where $\delta$ represents the variational operator.

${ }^{2}$ Similar functionals may be included for each interface if it is desired to examine delamination (debonding of the constituent layers) of the patch itself. We are here interested in debonding of the patch from the base structure, hence such terms are excluded for brevity and clarity of presentation.
} 
pressure. (For the case of a flat structure, the dimensional radius $\bar{R}$ is replaced by the dimensional half length $\bar{L}_{0}$ of the base structure, in each of the above normalizations.)

The Principle of Stationary Potential Energy, in the present context, may be stated as

$$
\delta \Pi=0,
$$

where $\delta$ corresponds to the variational operator.

Taking the appropriate variations, allowing the interior boundaries $a$ and $b$ to vary along with the displacements, we arrive at the corresponding differential equations, boundary and matching conditions, and transversality conditions. (The transversality conditions establish values of the variable interior boundaries $a$ and $b$, to be found as part of the solution together with the associated displacement field, which correspond to equilibrium configurations of the evolving structure.) After eliminating the Lagrange multipliers from the resulting equations, we arrive at a selfconsistent set of equations, and conditions (including energy release rates) for the evolving composite structure. We thus have

$$
\begin{aligned}
& M_{i}^{* \prime \prime}+k\left(M_{i}^{*}-N_{i}^{*}\right)-\left(N_{i}^{*} w_{i}^{* \prime}\right)^{\prime}=-p_{0}, \quad N_{i}^{* \prime}=0, \quad\left(s \in S_{i} ; i=1,2\right) \\
& M_{3}^{\prime \prime}+k\left(M_{3}-N_{3}\right)-\left(N_{3} w_{3}^{*^{\prime}}\right)^{\prime}=-p_{0}, \quad N_{3}^{\prime}=0, \quad\left(s \in S_{3}\right) \\
& M_{3}^{\circ \prime \prime}+k\left(M_{3}^{\circ}-N_{3}^{\circ}\right)-\left(N_{3}^{\circ} w_{3}^{\circ}\right)^{\prime}=0, \quad N_{3}^{\circ}=0, \quad\left(s \in S_{3}\right)
\end{aligned}
$$

with

$$
\begin{aligned}
& w_{i}^{\circ}(s) \equiv w_{1 i}(s)=w_{2 i}(s)=\cdots=w_{q i}(s), \quad\left(s \in S_{i} ; i=1,2,3 ; q \leqslant n\right) \\
& u_{i}^{\circ}(s) \equiv u_{1 i}+\rho_{1} w_{i}^{\circ \prime}=u_{2 i}+\rho_{2} w_{i}^{\circ \prime}=\cdots=u_{q i}+\rho_{q} w_{i}^{\circ \prime} \quad\left(s \in S_{i} ; i=1,2,3 ; q \leqslant n\right) \\
& w_{i}^{*}(s) \equiv w_{0 i}(s)=w_{i}^{\circ}(s), \quad\left(s \in S_{i} ; i=1,2\right) \\
& \kappa_{i}^{*}(s) \equiv \kappa_{0 i}(s)=\kappa_{i}^{\circ}(s), \quad\left(s \in S_{i} ; i=1,2\right) \\
& u_{1}^{*}(s)=u_{1}^{\circ}(s), \quad\left(s \in S_{1}\right)
\end{aligned}
$$

where $u_{i}^{\circ}(s), w_{i}^{\circ}(s), e_{i}^{\circ}(s), \kappa_{i}^{\circ}(s)$ are the normalized circumferential (in-plane) and transverse displacements, membrane strain and curvature change, respectively, of the patch at the reference surface, while $u_{i}^{*}(s), w_{i}^{*}(s), e_{i}^{*}(s)$ and $\kappa_{i}^{*}(s)$ correspondingly refer to the displacements, membrane strain and curvature change of the base structure at the reference surface. Further,

$$
\begin{aligned}
N_{i}^{\circ}(s) & \equiv \sum_{\alpha=1}^{q \leqslant n} N_{\alpha i}(s)=C_{(q)}^{\circ} e_{i}^{\circ}(s)+B_{(q)}^{\circ} \kappa_{i}^{\circ}(s), \quad\left(s \in S_{i} ; i=1,2,3\right) \\
M_{i}^{\circ}(s) & \equiv \sum_{\alpha=1}^{q \leqslant n}\left\{D_{\alpha} \kappa_{\alpha i}(s)+\rho_{\alpha} N_{\alpha i}(s)\right\} \\
& =A_{(q)}^{\circ} \kappa_{i}^{\circ}(s)+B_{(q)}^{\circ} e_{i}^{\circ}(s)=D_{(q)}^{\circ} \kappa_{i}^{\circ}(s)+\rho_{(q)}^{\circ} N_{i}^{\circ}, \quad\left(s \in S_{i} ; i=1,2,3\right)
\end{aligned}
$$

correspond to the normalized resultant membrane force and resultant bending moment (about an axis through the reference surface), respectively, of the entire patch cross section in region $S_{i}$, where the stiffnesses of the composite patch are found in terms of the stiffnesses of the individual layers as 


$$
\begin{aligned}
& A_{(q)}^{\circ}=\sum_{\alpha=1}^{q \leqslant n}\left\{D_{\alpha}+\rho_{\alpha}^{2} C_{\alpha}\right\}, \quad B_{(q)}^{\circ}=\sum_{\alpha=1}^{q \leqslant n} \rho_{\alpha} C_{\alpha}, \quad C_{(q)}^{\circ}=\sum_{\alpha=1}^{q \leqslant n} C_{\alpha}, \\
& D_{(q)}^{\circ}=A_{(q)}^{\circ}-\rho_{(q)}^{\circ} B_{(q)}^{\circ}, \quad \text { and } \quad \rho_{(q)}^{\circ}=B_{(q)}^{\circ} / C_{(q)}^{\circ} .
\end{aligned}
$$

The parameter $D_{(q)}^{\circ}$ is seen to correspond to the bending stiffness of the composite patch measured with respect to its neutral axis at coordinate $s$, while the parameter $\rho_{(q)}^{\circ}$ is seen to locate the neutral axis of the composite patch with respect to the reference surface at this coordinate.

For the isotropic base structure we have simply that

$$
N_{i}(s) \equiv N_{0 i}(s) \quad \text { and } \quad M_{i}(s) \equiv D_{0} \kappa_{0 i}(s)-\frac{h_{0}}{2} N_{0 i}(s), \quad\left(s \in S_{i} ; i=1,2,3\right)
$$

where $N_{i}(s)$ and $M_{i}(s)$, respectively, correspond to the normalized resultant membrane force and bending moment acting on the cross section of the base panel, measured with respect to the reference surface, in region $S_{i}$. (For a layered base structure, $N_{i}(s)$ and $M_{i}(s)$ would be of the same general form as the corresponding parameters for the patch but without the effect of the taper, i.e. $q \neq q(s)$ - the number of layers being uniform throughout the span.) In a manner similar to that for the composite patch, we have for the composite structure (patch + base panel),

$$
\begin{aligned}
N_{1}^{*}(s) & \equiv N_{1}+N_{1}^{\circ}=C_{(q)}^{*} e_{1}^{*}(s)+B_{(q)}^{*} \kappa_{1}^{*}(s), \\
M_{1}^{*}(s) & \equiv M_{1}(s)+M_{1}^{\circ}(s) \\
& =A_{(q)}^{*} \kappa_{1}^{*}(s)+B_{(q)}^{*} e_{1}^{*}(s)=D_{(q)}^{*} \kappa_{1}^{*}(s)+\rho_{(q)}^{*} N_{1}^{*},
\end{aligned}
$$

where $N_{1}^{*}(s)$ and $M_{1}^{*}(s)$, respectively, correspond to the normalized membrane force and normalized bending moment in the bonded portion of the composite structure (patch + base panel), and

$$
\begin{aligned}
& N_{2}^{*}(s) \equiv N_{2}+N_{2}^{\circ}, \\
& M_{2}^{*}(s) \equiv M_{2}(s)+M_{2}^{\circ}(s)=\mu_{(q)}^{*} \kappa_{2}^{*}(s)+\left(\rho_{(q)}^{\circ} N_{2}^{\circ}-\frac{h_{0}}{2} N_{02}\right),
\end{aligned}
$$

respectively, correspond to the normalized resultant membrane force and bending moment of the debonded portion of the composite structure in the contact zone.

The stiffnesses of the composite structure defined by eqns (20a), (20b) and (21b) are found, in terms of the stiffnesses and thicknesses of the substructures, as

$$
\begin{aligned}
& A_{(q)}^{*}=D_{0}+\left(h_{0} / 2\right)^{2} C_{0}+A_{(q)}^{\circ}, \\
& B_{(q)}^{*}=B_{(q)}^{\circ}-\left(h_{0} / 2\right) C_{0}, \\
& C_{(q)}^{*}=C_{0}+C_{(q)}^{\circ}, \\
& D_{(q)}^{*}=A_{(q)}^{*}-\rho_{(q)}^{*} B_{(q)}^{*}, \\
& \rho_{(q)}^{*}=B_{(q)}^{*} / C_{(q)}^{*} . \\
& \mu_{(q)}^{*}=D_{0}+D_{(q)}^{\circ} .
\end{aligned}
$$

The parameter $D_{(q)}^{*}$ is seen to correspond to the bending stiffness of the composite structure 
measured with respect to its neutral axis at coordinate $s$, while the parameter $\rho_{(q)}^{*}$ is seen to (radially/transversely) locate the neutral axis of the composite structure with respect to the reference surface at this coordinate in the bond zone. The parameter $\mu_{(q)}^{*}$ may be identified as the bending stiffness of the composite structure with respect to its neutral axis at coordinate $s$, in the contact zone.

The associated boundary and matching conditions obtained similarly take the forms:

$$
u_{1}^{*}(0)=0, \quad w_{1}^{* \prime}(0)=0,
$$

and

$$
\left[M_{1}^{* \prime}-N_{1}^{*} w_{1}^{* \prime}\right]_{s=0}=0 \quad \text { (applied pressure or circumferential/in-plane tension) }
$$

or

$$
\begin{aligned}
& w_{1}^{*}(0)=0, \quad(\text { three-point loading) } \\
& u_{1}^{*}(a)=u_{2}^{*}(a)=u_{2}^{\circ}(a), \quad N_{1}^{*}(a)=N_{2}^{*}(a), \\
& w_{1}^{*}(a)=w_{2}^{*}(a), \quad w_{1}^{* \prime}(a)=w_{2}^{* \prime}(a), \\
& M_{1}^{*}(a)=M_{2}^{*}(a), \quad\left[M_{1}^{* \prime}-N_{1}^{*} w_{1}^{* \prime}\right]_{s=a}=\left[M_{2}^{* \prime}-N_{2}^{*} w_{2}^{* \prime}\right]_{s=a}, \\
& u_{2}^{*}(b)=u_{3}^{*}(b), \quad N_{2}(b)=N_{3}(b), \\
& u_{2}^{\circ}(b)=u_{3}^{\circ}(b), \quad N_{2}^{\circ}(b)=N_{3}^{\circ}(b), \\
& w_{2}^{*}(b)=w_{3}^{*}(b)=w_{3}^{\circ}(b), \\
& w_{2}^{* \prime}(b)=w_{3}^{* \prime}(b)=w_{3}^{\circ}(b), \\
& M_{2}^{*}(b)=M_{3}(b)+M_{3}^{\circ}(b), \\
& {\left[M_{2}^{* \prime}-N_{2}^{*} w_{2}^{* \prime}\right]_{s=b}=\left[M_{3}^{\prime}-N_{3} w_{3}^{* \prime}\right]_{s=b}+\left[M_{3}^{\circ \prime}-N_{3}^{\circ} w_{3}^{\circ \prime}\right]_{s=b},} \\
& N_{3}^{\circ}\left(L_{1}\right)=\kappa_{3}^{\circ}\left(L_{1}\right)=\left[M_{3}^{\circ \prime}-N_{3}^{\circ} w_{3}^{\circ}\right]_{s=L_{1}}=0, \\
& u_{03}\left(L_{0}\right)=0 \quad \text { or } N_{03}\left(L_{0}\right)=T_{0} \quad\left(T_{0} \text { prescribed }\right),
\end{aligned}
$$

and

$w_{03}\left(L_{0}\right)=0, \quad$ and $\quad w_{03}^{\prime}\left(L_{0}\right)=0 \quad$ or $\kappa_{03}\left(L_{0}\right)=0, \quad$ (applied pressure and tension)

$\left(26 \mathrm{~b}, \mathrm{c}, \mathrm{c}^{\prime}\right)$

or

$$
\left[M_{3}^{\prime}-N_{3} w_{3}^{\prime}\right]_{s=L_{0}}=-Q_{0} \quad\left(Q_{0} \text { prescribed) } \quad \text { and } \quad \kappa_{03}\left(L_{0}\right)=0 . \quad\right. \text { (3-point loading) }
$$

Conditions similar to those defined by eqns (23a)-(23g) or (24a)-(24j) must also be imposed at the boundary of each step (i.e., at $s=L_{\alpha} ; \alpha=1,2, \ldots, n$ ), the specific form depending upon whether the boundary in question occurs within the bond zone, contact zone, or lift zone. This occurs since the stiffnesses of the composite patch [see eqns $\left.\left(18^{\prime} \mathrm{a}-\mathrm{e}\right)\right]$ and hence also of the 
composite structure [see eqns $\left(20^{\prime} \mathrm{a}-\mathrm{e}\right)$ and $\left(21^{\prime}\right)$ ] are piecewise constant and hence generally not continuously differentiable within the tapered region of the patch. Hence,

$$
\begin{aligned}
& u_{1}^{*}\left(L_{q^{+}}\right)=u_{1}^{*}\left(L_{q^{-}}\right), \quad N_{1}^{*}\left(L_{q^{+}}\right)=N_{1}^{*}\left(L_{q^{-}}\right), \quad \forall L_{q} \in(0, a) \\
& w_{i}^{*}\left(L_{q^{+}}\right)=w_{i}^{*}\left(L_{q^{-}}\right), \quad w_{i}^{* \prime}\left(L_{q^{+}}\right)=w^{*_{i}^{\prime}}\left(L_{q^{-}}\right), \quad(i=1,2) \quad \forall L_{q} \in(0, b) \\
& M_{i}^{*}\left(L_{q^{+}}\right)=M_{i}^{*}\left(L_{q^{-}}\right), \quad(i=1,2) \quad \forall L_{q} \in(0, b) \\
& {\left[M_{i}^{* \prime}-N_{i}^{*} w_{i}^{* \prime}\right]_{s=L_{q^{+}}}=\left[M_{i}^{* \prime}-N_{i}^{*} w_{i}^{* \prime}\right]_{s=L_{q^{-}}}(i=1,2) \quad \forall L_{q} \in(0, b)} \\
& u_{i}^{\circ}\left(L_{q^{+}}\right)=u_{i}^{\circ}\left(L_{q^{-}}\right), \quad N_{i}^{\circ}\left(L_{q^{+}}\right)=N_{i}^{\circ}\left(L_{q^{-}}\right), \quad(i=2,3) \quad \forall L_{q} \in\left(a, L_{1}\right) \\
& w_{3}^{\circ}\left(L_{q^{+}}\right)=w_{3}^{\circ}\left(L_{q^{-}}\right), \quad w_{3}^{\circ \prime}\left(L_{q^{+}}\right)=w_{3}^{\circ}\left(L_{q^{-}}\right), \quad \forall L_{q} \in\left(b, L_{1}\right) \\
& M_{3}^{\circ}\left(L_{q^{+}}\right)=M_{3}^{\circ}\left(L_{q^{-}}\right), \quad \forall L_{q} \in\left(b, L_{1}\right) \\
& {\left[M_{3}^{\circ}-N_{3}^{\circ} w_{3}^{\circ}\right]_{s=L_{q^{+}}}=\left[M_{3}^{\circ}-N_{3}^{\circ} w_{3}^{\circ \prime}\right]_{s=L_{q^{-}}} \quad \forall L_{q} \in\left(b, L_{1}\right)}
\end{aligned}
$$

This situation is alleviated somewhat for the case where the taper is continuous. For the case of $a$ continuous end taper, the above conditions are satisfied identically (stiffnesses, etc. are differentiable), except at $s=L_{1}$ and $s=L_{n}$.

The transversality condition for the propagating bond zone boundary, $s=a$, takes the following forms depending upon the presence or absence of a contact zone. Hence,

$$
\begin{aligned}
\mathscr{G}_{\mathscr{A}}\{a\} \equiv\left[\frac{1}{2} \mu_{(q)}^{*} \kappa_{2}^{* 2}+\frac{1}{2 C_{0}} N_{02}^{2}+\frac{1}{2 C_{(q)}^{\circ}} N_{2}^{\circ 2}\right]_{s=a^{\circ}} \\
-\left[\frac{1}{2} D_{(q)}^{*} \kappa_{1}^{* 2}+\frac{1}{2 C_{(q)}^{*}} N_{1}^{* 2}\right]_{s=a}=2 \gamma, \quad\left(b \geqslant a^{+}\right) \\
\mathscr{G}_{\mathscr{B}}\{a\} \equiv\left[\frac{1}{2} D_{0} \kappa_{03}^{2}+\frac{1}{2} D_{(q)}^{\circ} \kappa_{3}^{\circ 2}+\frac{1}{2 C_{0}} N_{03}^{2}+\frac{1}{2 C_{(q)}^{\circ}} N_{3}^{\circ 2}\right]_{s=a} \\
-\left[\frac{1}{2} D_{(q)}^{*} \kappa_{1}^{* 2}+\frac{1}{2 C_{(q)}^{*}} N_{1}^{* 2}\right]_{s=a}=2 \gamma, \quad(b=a)
\end{aligned}
$$

where $\mathscr{G}_{\mathscr{A}}\{a\}$ and $\mathscr{G}_{\mathscr{B}}\{a\}$ are identified as the energy release rates. The conditions (29) suggest the following delamination criterion:

if, for some initial value of $a=a_{0}$, we have that $\mathscr{G}\left\{a_{0}\right\} \geqslant 2 \gamma$, then debonding occurs and the system evolves (a decreases, $a^{*}$ increases) such that the corresponding equality (29a) or (29b) is satisfied. If $\mathscr{G}\left\{a_{0}\right\}<2 \gamma$, debonding does not occur.

For a propagating contact zone boundary $s=b$, the associated transversality condition reduces to the form

$$
\left[\frac{1}{2} D_{0} \kappa_{03}^{2}+\frac{1}{2} D_{(q)}^{\circ} \kappa_{3}^{\circ 2}\right]_{s=b^{+}}-\left[\frac{1}{2} \mu_{(q)}^{*} \kappa_{2}^{* 2}\right]_{s=b^{-}}+\frac{N_{3}^{\circ 2}}{2}\left[\left.\frac{1}{C_{(q)}^{\circ}}\right|_{s=b^{+}}-\left.\frac{1}{C_{(q)}^{\circ}}\right|_{s=b^{-}}\right]=0 .
$$


It is seen that for continuous tapering the jump in membrane energy density across the contact zone-lift zone boundary (the last bracket on the left hand side) vanishes identically. Hence, incorporating the corresponding moment balance given by eqn (24i), it is seen that for such tapering eqn (30) reduces to the condition that

$$
\kappa_{2}^{*}(b)=\kappa_{03}(b)=\kappa_{3}^{\circ}(b), \quad\left(b<L_{1}\right)
$$

to which we add the qualification

$$
\kappa_{03}\left(b^{+}\right)>\kappa_{3}^{\circ}\left(b^{+}\right)
$$

to prohibit penetration of the base panel and patch for $s \in S_{3}$. (It will be seen shortly that eqn (30) reduces to a statement of continuity of curvature change at the contact zone boundary for the discrete case as well, for the class of problems of interest.) It is thus seen that such a boundary is defined by the point where the curvature changes of the respective segments of the structure become continuous. The system (1)-(30) defines the class of problems of interest.

The boundary conditions (25), together with eqns $(15 \mathrm{a}, \mathrm{b})$, indicate that the 'flap' (i.e., the segment of the debonded portion of the patch which is lifted away from the base structure) is unloaded and hence that

$$
N_{3}^{\circ}(s)=\kappa_{3}^{\circ}(s)=M_{3}^{\circ}(s)=0 \quad\left(\forall s \in S_{3}\right) .
$$

Further, integration of eqns (13b), (14b) and (15b), imposition of the associated matching conditions (23c), (24b), and (24d), and incorporation of eqn (31a) while recalling eqn (21a) yields the results

$$
N_{1}^{*}=N_{2}=N_{3}=N_{0}=\text { constant }, \quad N_{3}^{\circ}=N_{2}^{\circ}=0 .
$$

The remaining equations and conditions are modified accordingly, with the transversality conditions (29)-(30) taking the forms

$$
\begin{aligned}
& \mathscr{G}_{\mathscr{A}}\{a\} \rightarrow\left[\frac{1}{2} \mu_{(q)}^{*} \kappa_{2}^{* 2}-\frac{1}{2} D_{(q)}^{*} \kappa_{1}^{* 2}+\frac{1}{2 C_{(q)}^{(e)}} N_{0}^{2}\right]_{s=a}=2 \gamma, \quad\left(b \geqslant a^{+}\right) \\
& \mathscr{G}_{\mathscr{B}}\{a\} \rightarrow\left[\frac{1}{2} D_{0} \kappa_{03}^{2}-\frac{1}{2} D_{(q)}^{*} \kappa_{1}^{* 2}+\frac{1}{2 C_{(q)}^{(e)}} N_{0}^{2}\right]_{s=a}=2 \gamma, \quad(b=a)
\end{aligned}
$$

and

$$
\kappa_{2}^{*}(b)=\kappa_{03}(b)=0, \quad \kappa_{03}\left(b^{+}\right)>0, \quad\left(b<L_{1}\right)
$$

regardless of whether the tapering is continuous or discrete, where

$$
1 / C_{(q)}^{(e)} \equiv \frac{\left\{C_{(q)}^{\circ} / C_{0}\right\}}{C_{(q)}^{*}} .
$$

It may be seen from eqn $\left(30^{\prime \prime}\right)$ that a propagating, or intermediate, contact zone boundary may occur only if conditions are such that an inflection point occurs in the interval $a<s<L_{1}$. If not, the system will possess either a full contact zone $\left(b=L_{1}\right)$, or no contact zone $(b=a)$. For the former case, the lifted segment of the flap (region $S_{31}$ ) will not exist, and the condition 


$$
\kappa_{2}^{*}(s)<0 \quad\left(a \leqslant s \leqslant b=L_{1}\right)
$$

must be satisfied (see Section 3.1). If $b=a$, then the condition of 'edge point contact', the situation in which only the free edge of the composite patch maintains sliding contact with the base structure, is possible. (For the purposes of brevity and clarity of presentation, the formulation for this case is presented separately in Appendix B.) Alternatively, $b=a$ and no contact at all occurs between the debonded segments of the patch and the base structure.

Integrating the strain-displacement relations and imposing the corresponding boundary and matching conditions for the circumferential displacements results in the integrability condition given by

$$
\begin{aligned}
u_{03}\left(L_{0}\right)= & N_{0}\left[\frac{a^{*}}{C_{0}}+\frac{a}{C_{\left(q^{\circ}\right)}^{*}}\right]+N_{0}\left\{\sum_{p=q^{\circ}+1}^{n} L_{(p)}\left[\frac{1}{C_{(p)}^{*}}-\frac{1}{C_{(p-1)}^{*}}\right]\right\} \\
& -\left[\frac{h_{0}}{2}+\rho_{\left(q^{\circ}\right)}^{*}\right] w_{01}^{\prime}(a)-\left\{\sum_{p=q^{\circ}+1}^{n}\left[\rho_{(p)}^{*}-\rho_{(p-1)}^{*}\right] w_{1}^{\prime}\left(L_{(p)}\right)\right\} \\
& +\sum_{i=1}^{3} \int_{S_{i}}\left[k\left(1-\rho_{(q)}^{*} \delta_{i 1}\right) w_{0 i}-\frac{1}{2} w_{0 i}^{\prime 2}\right] \mathrm{d} s,
\end{aligned}
$$

where $\delta_{i j}$ is Kronecker's delta, and $q^{\circ} \equiv q(a)$. We are reminded that $1 \leqslant q(s) \leqslant n$ and hence that $\rho_{(q)}^{*}$ appearing in the integrand is piecewise constant over $S_{1}$. The counterparts of eqns (13a) and (14a) and the corresponding boundary and matching conditions obtained upon substitution of the results (31) and (32), together with the transversality conditions $\left(29^{\prime}\right)$ and $\left(30^{\prime \prime}\right)$, and the integrability condition (34), transform the problem statement into a mixed formulation in terms of the transverse displacement $w_{0 i}(s)(i=1-3)$, the membrane force $N_{0}$, and the propagating boundaries $a$ and $b$. A discussion pertaining to the analysis of the problems of the class of interest is presented in the next section.

\section{Analysis}

In this section the basis of a linear analysis of the problems of interest is presented. The analysis is based on the general formulation presented in the previous section. As in (Bottega, 1995) we first examine the existence and characteristics of a contact zone, and then outline the analysis for an evolving structure of the class under consideration. It will be shown that a contact zone can exist for the case of a pressure loaded structure (plate or shell) for the situation where the supports are such that rotation at the edge is prohibited (clamped edges). It will further be shown that when a contact zone does exist then it will traverse the entire debonded region of the patch (i.e., it is a 'full' contact zone). The arguments and characteristics follow the same general form as in (Bottega, 1995), for flat plates with untapered patches. Such characteristics were observed numerically for patched shells without taper (Bottega and Loia, 1996), but an analytical proof for this class of problems was not given therein. This was also the case for patched circular plates without taper (Bottega and Loia, 1997). 


\subsection{Contact zone}

Upon substitution of the linearized version of eqn (13a) into the corresponding equation for the patch in region 2 (not presented), we arrive at an expression for the associated (transversely directed) interfacial stress $\sigma_{1}^{(2)}$ given by

$$
\sigma_{1}^{(2)}(s)=-\frac{D_{(q)}^{\circ}}{\mu_{(q)}^{*}} p_{\text {eff }} \leqslant 0,
$$

where

$$
p_{\text {eff }} \equiv p_{0}-k N_{0} \geqslant 0, \quad(k=0,1)
$$

and we recall that $k=0$ corresponds to the case of a flat structure and $k=1$ corresponds to the case of a cylindrical structure. It can be seen from eqn (35a) that the interfacial stress is piecewise constant throughout the contact zone (though it may not be uniform due to the presence of the steps of the patch). As indicated in eqns $(35 \mathrm{a}, \mathrm{b})$, we stipulate that when contact between the debonded surfaces of the base panel and the patch occurs, the contact stress must necessarily be compressive, i.e. that $\sigma_{1}^{(2)} \leqslant 0\left(p_{\text {eff }} \geqslant 0\right)$. Upon consideration of eqns (35a) and (35b), it may be seen that $\sigma_{1}^{(2)} \geqslant 0$ when $p_{0}=0\left(k N_{0} \geqslant 0\right)$, from which it may be concluded that a contact zone does not exist for the cases under consideration for which the applied pressure vanishes (the exception being the case of passive contact, $\kappa_{2}^{*}(s)=\kappa_{03}(s)=0$, for the tensile loaded plate with pinned supports - see Bottega, 1995). For situations where the pressure is nonvanishing, the existence and characteristics of a contact zone will be dependent upon the characteristics of the curvature changes occurring in the various regions of the structure.

Upon solution of the governing equations presented in the previous section, it is found that the curvature changes in each segment are of the forms given by

$$
\kappa_{1}^{*}(s)=\frac{f(s)-N_{0}\left(\rho_{(q)}^{*}+\frac{h}{2}\right)}{D_{(q)}^{*}}, \quad \kappa_{2}^{*}(s)=\frac{f(s)}{\mu_{(q)}^{*}}, \quad \kappa_{3}^{*}(s)=\frac{f(s)}{D_{0}},
$$

where

$$
f(s)= \begin{cases}c_{0}-\frac{1}{2} p_{0} s^{2} & (k=0) \\ c_{1} \cos (s)-p_{\text {eff }} & (k=1)\end{cases}
$$

and the constants $c_{0}$ and $c_{1}$ depend upon the support conditions for the specific problem of interest (for specific values of the lift zone - contact zone boundary, $b$, and the bond zone boundary, $a$ ). It is seen from eqns $(36 \mathrm{~b}, \mathrm{c})$ and $(37 \mathrm{a}, \mathrm{b})$ that the debonding structure possesses at most one inflection point on the interval $s \in[a, 1]$ for a flat structure or $s \in[a, \pi]$ for a cylindrical structure. By virtue of eqns $\left(30^{\prime \prime} \mathrm{a}, \mathrm{b}\right)$, this point will correspond to the bond zone boundary $s=b$ provided it does not occur beyond the domain of definition of the patch and provided eqn $\left(30^{\prime \prime} \mathrm{c}\right)$ is satisfied.

Consideration of a free body diagram of the debonded segments of the patch indicates that

$$
\kappa_{2}^{*}(s) \leqslant 0, \quad s \in(a, b)
$$

for contact. It may be easily shown via eqns $(37 \mathrm{a}, \mathrm{b})$ that for the case of hinged supports (free or 
fixed) $\kappa^{*}(s)>0$ for all $s>a$, for both the flat $(k=0)$ and cylindrical $(k=1)$ classes of structures under consideration. Since this contradicts eqn (38), it follows that no contact zone exists for these cases.

For the case of clamped support conditions we make the following argument. If eqn (38) is satisfied, it follows from eqns $(36 \mathrm{~b}, \mathrm{c})$ and $(37 \mathrm{a}, \mathrm{b})$ that $\kappa_{3}^{*}(s)<0$. This violates the condition given by eqn $\left(30^{\prime \prime} \mathrm{c}\right)$. Thus, if a contact zone occurs under these conditions, it will be a 'full contact zone' (that is, the entire debonded segment of the patch will maintain contact with the base structure). Alternatively, no contact zone will exist if $\kappa_{2}^{*}\left(a^{+}\right)<0$. We thus see that for the pressure loaded structure (flat or curved), the composite structure possesses either no contact zone or a full contact zone, but the occurrence and behavior of a propagating contact zone is not an issue. These conditions are incorporated into the analysis outlined in the remainder of this section, to predict the behavior of the evolving system.

\subsection{Evolving structure}

Paralleling the analyses of Bottega (1995), Bottega and Loia (1996) and Bottega and Loia (1997) for similarly configured structures without taper, we first define the normalized loading parameter $\lambda$ and characteristic deflection $\Delta$, for each of the specific problems (applied tension, three-point transverse loading, and applied pressure), respectively, as follows :

$$
\left\{\lambda=T_{0}, \Delta=u_{03}\left(L_{0}\right)\right\} ; \quad\left\{\lambda=Q_{0}, \Delta=w_{03}\left(L_{0}\right)\right\} ; \quad\left\{\lambda=p_{0}, \Delta=-w_{01}(0)\right\} .
$$

We shall also define the 'global stiffness' for each particular problem as

$$
K \equiv \lambda / \Delta
$$

for each $(\lambda, \Delta)$ pair defined in eqn (39).

Since we shall perform a linear analysis, the response in each case will be found to be proportional to the loading parameter for the specific problem under consideration. The integrability condition, eqn (34), will then take the general form

$$
u_{03}\left(L_{0}\right)=\lambda \mathscr{F}_{\lambda}(a)+N_{0} \mathscr{F}_{N}(a),
$$

where $\mathscr{F}_{\lambda}(a)$ and $\mathscr{F}_{N}(a)$ are functions obtained by substituting the specific analytical solution for the transverse displacement into eqn (34). For problems where $\lambda=T_{0}$, we have, from condition (26a'), that $N_{0}=T_{0}$. Equation (41) then gives the normalized in-plane/circumferential edge displacement as a function of the applied tension for this case. For other loading types, eqn (41) gives the normalized membrane force $N_{0}$ as a function of $\lambda$ for fixed end conditions $\left(u\left(L_{0}\right)=0\right)$, and gives the in-plane/circumferential edge displacement as a function of $\lambda$ for free edge conditions, where $N_{0}=0$ from eqn $\left(26 \mathrm{a}^{\prime}\right)$.

For each case and for each increment in load, the particular equilibrium configuration of the evolving system has associated with it a particular value of the contact zone boundary $b$ for a given value of $a$. It was established in the previous subsection that, within the context of the model, no contact zone is present $(b=a)$ for the case of applied in-plane/circumferential tension or for the case of three-point transverse loading. It was likewise established that the debonding structure will not possess a contact zone for the case of applied pressure when the base structure is supported such that its edges are free to rotate (hinged supports) whether free or fixed with regard to 
translation. For the case of internal pressure loading when the edges are prohibited from rotating (clamped supports), whether free or fixed with regard to translation, it was established that the presence of a full contact zone is possible. Thus, for this case either $b=a$, or $b=L_{0}$, the latter occurring only if the kinematic condition $\kappa_{2}^{*}\left(a^{+}\right)<0$ is satisfied. If this condition is not satisfied, then $b=a$.

With the above established, the energy release rates can be written in terms of the loading parameter explicitly, for each case under consideration. The equations for the growth paths/threshold curves $\lambda$ vs $a$ (or $a^{*}$ ) and $\Delta$ vs $a$ (or $a^{*}$ ) may then be found directly from the transversality conditions $\left(29^{\prime}\right)$, and take the general forms

$$
\lambda^{*} \equiv \lambda / \sqrt{2 \gamma}=1 / \sqrt{\Omega(a ; \mathbf{S}, b)}, \quad \Delta^{*} \equiv \Delta / \sqrt{2 \gamma}=K^{-1}(a ; \mathbf{S}, b) / \sqrt{\Omega(a ; \mathbf{S}, b)},
$$

where $b=a$ or $L_{1}, \Omega(a ; \mathbf{S}, b)$ is the normalized energy release rate per square of the normalized load, $\mathbf{S}$ is the set of stiffnesses of the structure, and $\left(\lambda^{*}, \Delta^{*}\right)$ correspond one to one with each $(\lambda, \Delta)$ pair defined earlier in this section. In this way, the evolution of the debonding structure may be characterized using the analytical solution for each particular problem of interest. Results for specific simple test configurations are presented in the next section. These include the tensile loaded patched plate with clamped-free and pinned-free edges and the three-point loaded patched plate specimen.

\section{Results for edge loaded plates}

In this section, results are presented for patched plates subjected to applied in-plane tension (Fig. 1(a)) and patched plates subjected to three-point transverse loading (Fig. 1(b)). In each case analytical solutions for the particular problem, based on a linearization of the formulation presented in Section 2, are employed to generate the corresponding threshold curves and stiffness degradation curves. We recall that for flat structures all length scales are normalized with respect to the half length of the base plate. We therefore consider the specific base structure of halfspan $L_{0}=1$. To provide direct comparison with corresponding results pertaining to plates with untapered patches (Bottega, 1995), we consider a base plate of normalized thickness $h_{0}=0.05$. We likewise consider a patch of the same total thickness $h_{p}=h_{0}$, outside the tapered region. In all cases, the patch under consideration will consist of a total of four identical layers $(n=4)$ outside the tapered region, hence $h_{\alpha}=h_{p} / n$ and $E_{\alpha}=E_{p}, \alpha=1,2, \ldots, n$, where we recall that $E_{\alpha}$ is the ratio of the modulus of layer $\alpha$ of the patch to that of the base plate, as defined in Section 2. It was shown in (Bottega, 1995) and related studies (Bottega and Loia, 1996) and (Bottega and Loia, 1997), where the characteristics of uniform untapered patches of modulus ratios of $0.1,1$, and 10 were studied and compared, that relatively compliant patches were generally more desirable than their stiffer counterparts with regard to the critical load level and the extent and stability of debonding. It is therefore of interest to examine the possibility that tapering the edge of the patch will suppress debonding, while maintaining a desired level of stiffness of the patch over the central region of the span. To this end then we shall consider an intermediately stiff patch, one for which the stiffnesses of the patch and hence the modulus of each of its layers is the same as that of the base plate. We thus consider patches such that $E_{\alpha}=E_{p}=1(\alpha=1-4)$ throughout this study. To investigate the influence of the degree of taper, simulations will be performed for a range of taper 
angles, $\psi$, where $\psi$ is defined as shown in Fig. 2. For brevity, results are presented for the range $60^{\circ} \leqslant \psi \leqslant 87^{\circ}$, as well as for the untapered case $\psi=0^{\circ}$, as these values of the taper angle are sufficient to demonstrate the effects of taper for these loading conditions. We recall from the corresponding discussion presented in Section 3 that a contact zone will not be present for the two loading types to be considered in this section. We first consider the case of applied in-plane tension.

\subsection{Patched plate subjected to applied in-plane tension}

We next present results for the case of a patched plate, such that the edges of the base plates are subjected to a distributed in-plane tensile load of normalized intensity $T_{0}$. Both hinged-free and clamped-free support conditions will be examined.

\subsubsection{Hinged-free supports}

The threshold curves and stiffness degradation curves for the case of hinged-free support conditions are displayed in Figs 4(a)-(c) for the specific taper angles, $\psi=60,80$ and $85^{\circ}$. The corresponding results for an untapered patch $\left(\psi=0^{\circ}\right)$ are also displayed in each figure for comparison. Specifically, the curves are presented in terms of the renormed tensile load, $T^{*}=T_{0} / \sqrt{2 \gamma}$, as a function of the conjugate bond zone size, $a^{*}$, in Fig. 4(a). In a similar fashion, the corresponding curves are presented in terms of the renormed (in-plane) edge deflection, $\Delta_{L}^{*}=u_{03}\left(L_{0}\right) / \sqrt{2 \gamma}$, as a function of $a^{*}$ in Fig. 4(b). The associated global stiffnesses, $K_{L}^{*}=T^{*} / \Delta_{L}^{*}$, are displayed as a function of $a^{*}$ in Fig. 4(c). It may be seen that each of the curves is piecewise continuous, with discontinuities occurring at the boundary of each particular step within the tapered region.

Consideration of Fig. 4(a) shows that the threshold curves expressed in terms of the critical tension are piecewise constant and hence that the tapering, in this case, simply raises the threshold value of the renormed tension above its base value (that for $\psi=0^{\circ}$ ) for a given reduction in patch thickness. The elevation in the threshold level of the load is seen to be the same throughout a segment of uniform patch thickness/stiffness, regardless of the taper angle and regardless of the location of the bond zone boundary. As was observed for the untapered case (Bottega, 1995), ${ }^{3}$ it is seen for the case of force controlled loading that once the critical load level is achieved debonding ensues and propagates in an unstable and catastrophic manner regardless of the initial size of debonded area.

Consideration of Fig. 4(b) shows the threshold curves expressed in terms of the edge deflection to be monotonically increasing within segments of constant patch thickness/stiffness. For this case however, the enhancement of the threshold level is seen to diminish with increased tapering for a given bond zone boundary though the elevation is, of course, spread out over the increased length of the step. It is seen that, for deflection controlled loading, debonding initially occurs in a stable

\footnotetext{
${ }^{3}$ We remark that for the patched plate only, for this particular loading case only and for this particular support condition only, the threshold curves for the untapered case presented in Bottega (1995) [and quoted in Loia and Bottega (1995)] are off by a numerical factor. The correct (constant) value corresponding to the modulus ratio $E_{\alpha}=1$ is $T^{*}=277$ as given above, while that corresponding to $E_{\alpha}=0.1$ is $T^{*}=459$ and that for $E_{\alpha}=10$ is $T^{*}=145$. We emphasize that this does not alter any of the qualitative results or corresponding conclusions presented in Bottega (1995) or Loia and Bottega (1995) pertaining to these or any other results.
} 
(a)

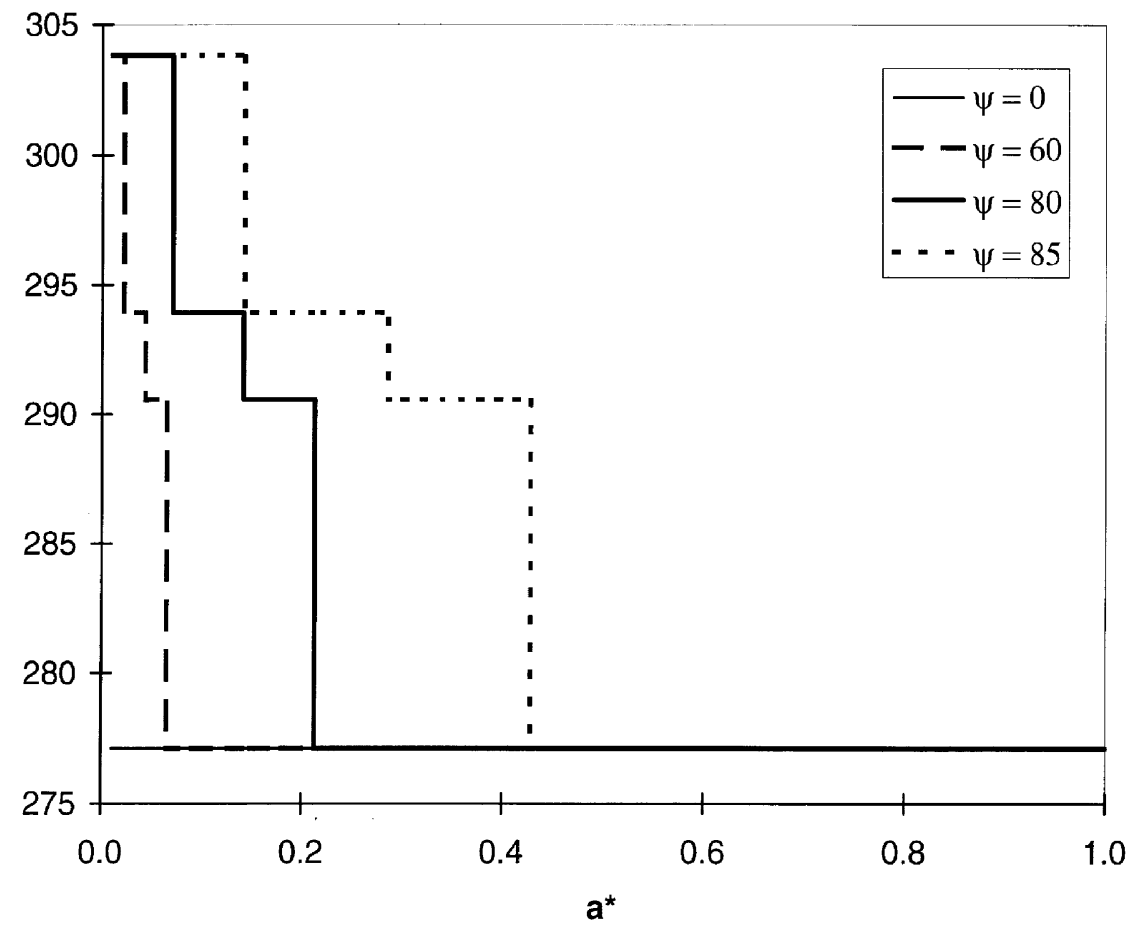

(b)

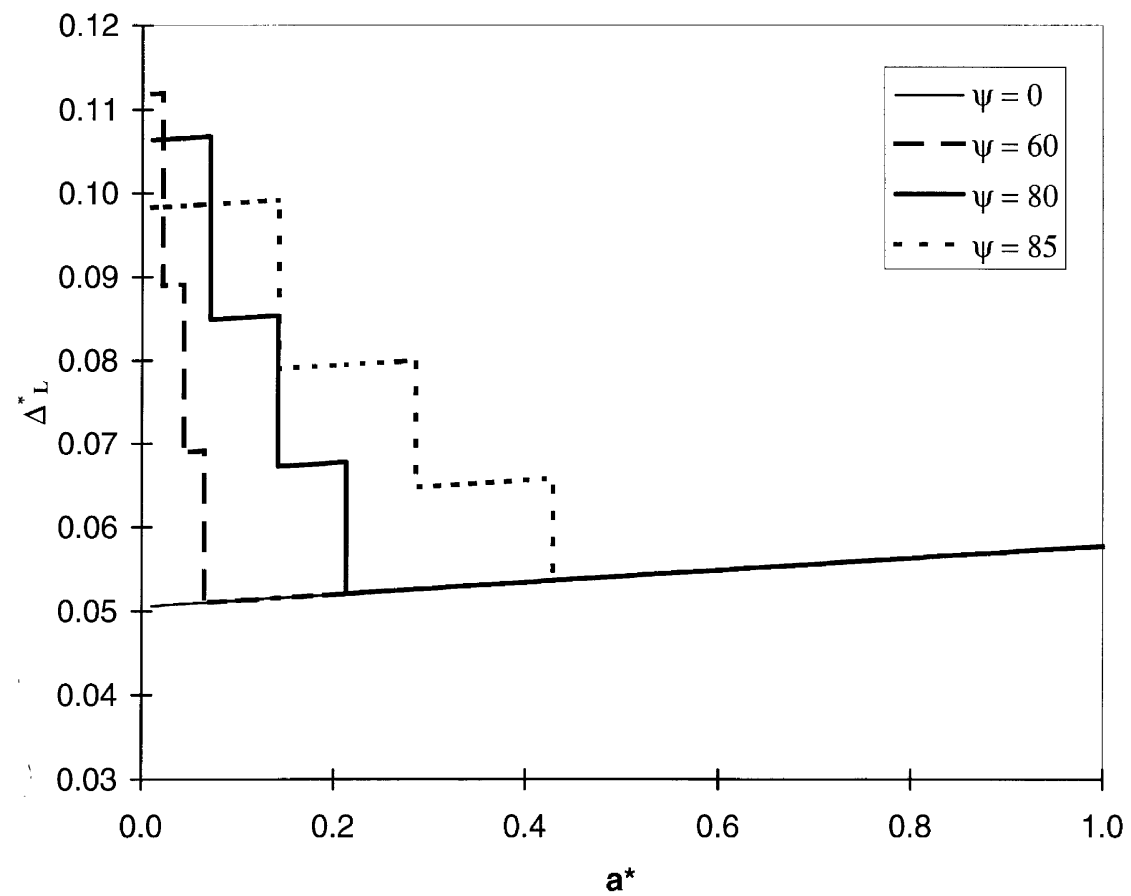

Fig. 4. Threshold curves for plates, with patches of selected taper angles, subjected to applied in-plane tension for the case of hinged-free supports. (a) Critical tension vs conjugate bond zone size, (b) critical in-plane edge deflection vs conjugate bond zone size, (c) associated global stiffness as a function conjugate bond zone size. 
(c)

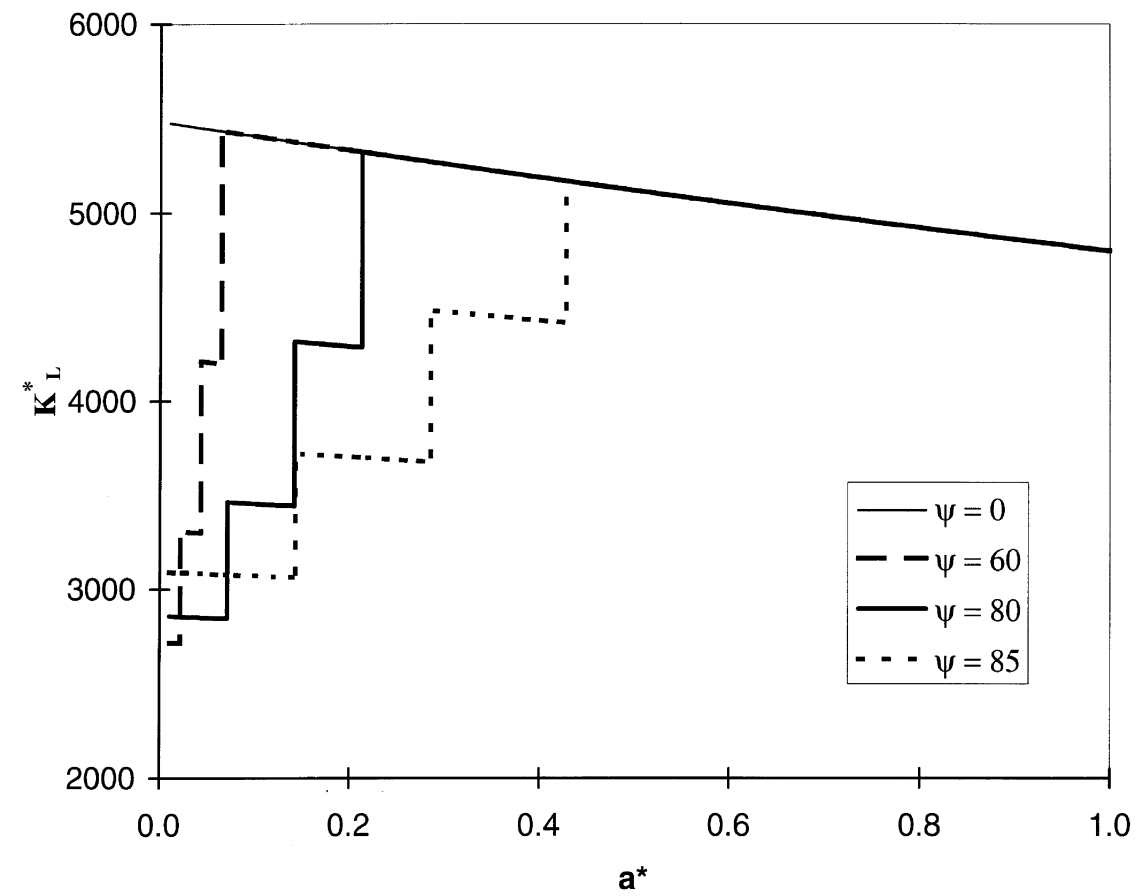

Fig. 4-continued.

manner for a given initial debond size but becomes unstable and catastrophic once the conjugate bond zone boundary encounters a step and that this may occur with only modest increases in the controlled edge deflection. In contrast, the untapered structure debonds in a stable manner regardless of the size of the debonded region. It is thus seen that for deflection controlled loading, tapering increases the critical 'load' level for debonding but can have catastrophic consequences if the critical level of the edge deflection is reached.

Finally, let us consider Fig. 4(c) which displays the reduction in global stiffness as a function of conjugate bond zone boundary. It may be noted that when the bond zone boundary lies within the tapered region the corresponding reduction in global stiffness is seen to be more dramatic, for a given step, as the taper angle diminishes. This seemingly counter intuitive result may be attributed to the fact that the more severe the taper, and hence the more flexible the structure, the larger the flexure and associated transverse deflection resulting from the difference in the location of the neutral axis within the bonded untapered region from that in the base plate. Such deformations tend to counter the in-plane stretching effects resulting in smaller in-plane deflections per unit load at the edges of the base plate and hence correspondingly higher values of the global stiffness.

\subsubsection{Clamped-free supports}

The threshold curves, expressed in terms of the renormed tension $T^{*}$ and renormed in-plane edge deflection $\Delta_{L}^{*}$, and the stiffness degradation curves for the case of clamped-free support conditions are, respectively, displayed in Figs 5(a)-(c) for the specific taper angles, $\psi=60,80$ and $85^{\circ}$, as well as for the untapered case $\psi=0^{\circ}$. It may be seen that each of the curves is piecewise 
(a)

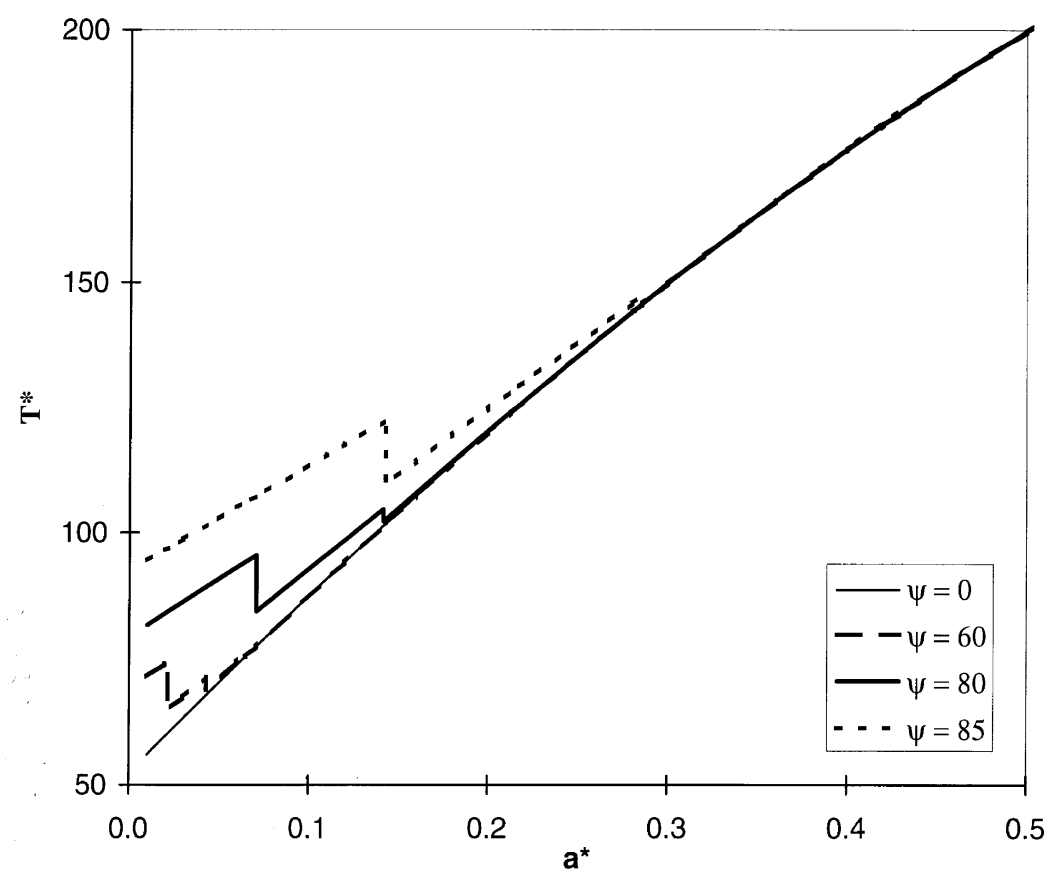

(b)

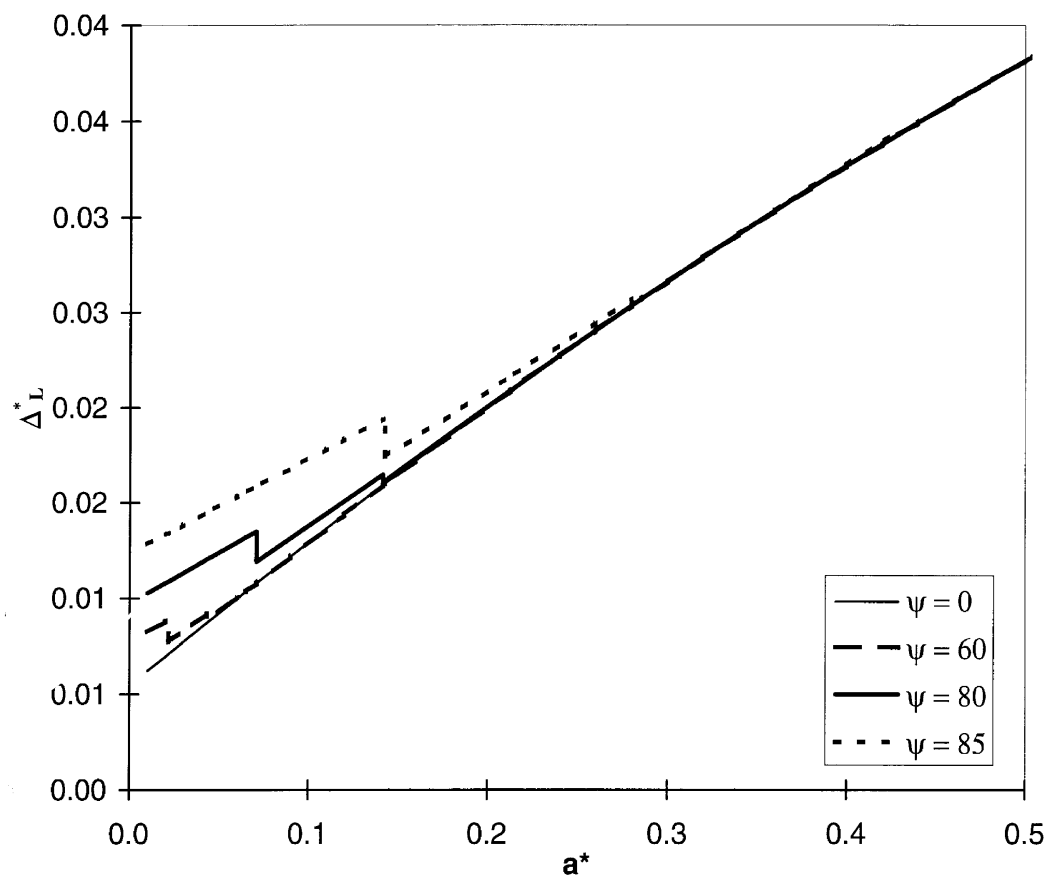

Fig. 5. Threshold curves for plates, with patches of selected taper angles, subjected to applied in-plane tension for the case of fixed-free supports. (a) Critical tension vs conjugate bond zone size, (b) critical in-plane edge deflection vs conjugate bond zone size, (c) associated global stiffness as a function conjugate bond zone size. 
(c)

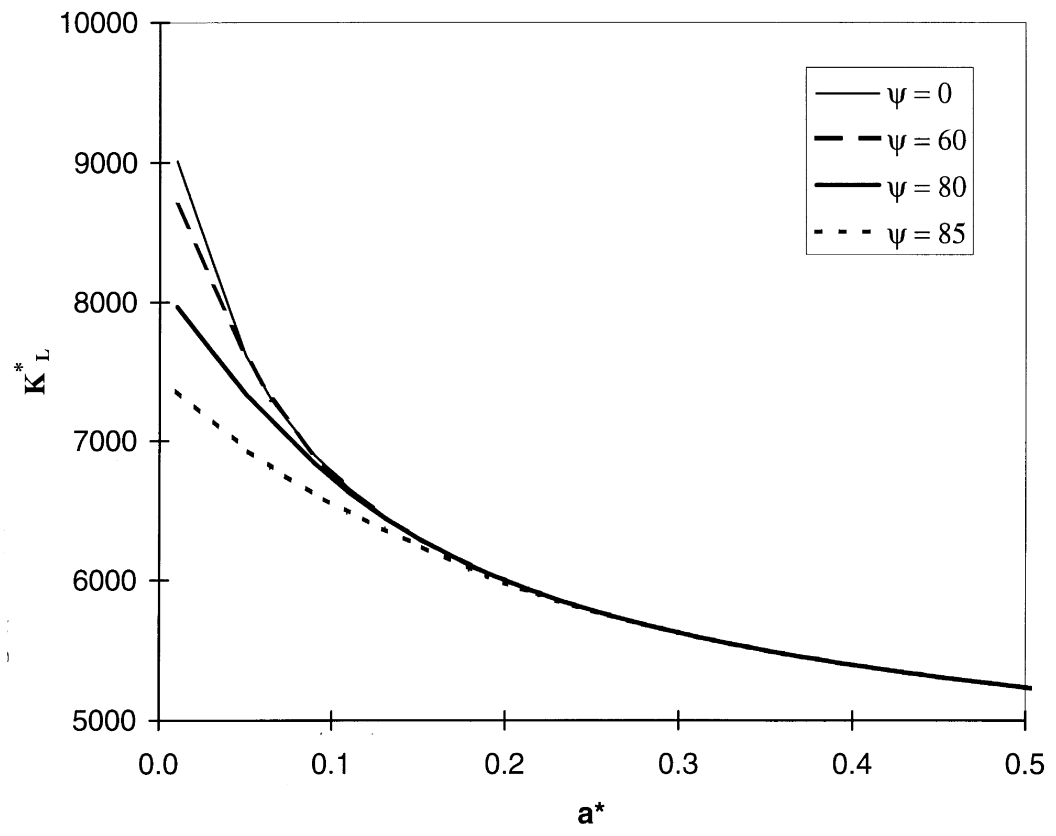

Fig. 5-continued.

continuous, with discontinuities occurring at the boundary of each particular step within the tapered region. The threshold curves depicted in both Figs 5(a) and (b) are seen to be monotonically increasing, within a segment of uniform patch thickness/stiffness, and to suddenly decrease at the boundary of each particular step. It may be further observed that increasing the degree of tapering raises the threshold level of both the applied tension and the in-plane edge deflection. The results therefore suggest, for both force and deflection controlled tests, that when the critical load level is achieved for a given initial bond size, debonding ensues and progresses in a stable manner until a step boundary is achieved at which point a moderate amount of unstable debonding occurs, followed by stable debonding and so on until the untapered region of the patch is encountered. At this point, debonding progresses in a stable manner as for the untapered case (Bottega, 1995). It may be noted that even when unstable debonding occurs, the extent of growth that is predicted for the present case is still below that which is predicted to occur for an untapered patch at the same load level. Finally, consideration of Fig. 5(c) indicates that the global stiffness is reduced with increased tapering when the bond zone boundary lies within the tapered region, as might be anticipated, as clamping of the edges restricts the amount of flexure and associated in-plane 'contraction' as compared with the case of hinged-free supports. For this particular loading and support condition, tapering of the edge of the patch may be considered to produce a favorable result in that the critical load levels are raised above those for the untapered patch and the extent of debonding is reduced even though some unstable debonding is predicted to occur where it would have been stable otherwise. 


\subsection{Patched plate subjected to three-point loading}

We next present results pertaining to the fundamental configuration of three-point loading of a patched plate whose edges are subjected to a distributed transverse load of normalized intensity $Q_{0}$. Threshold curves and stiffness degradation curves are displayed in Figs 6(a)-(c) for the specific taper angles, $\psi=80,85$ and $87^{\circ}$, as well as for the untapered case $\psi=0^{\circ}$. Specifically, threshold curves are presented in terms of the renormed load intensity, $Q^{*}=Q_{0} / \sqrt{2 \gamma}$, in Fig. 6(a) and the renormed transverse edge deflection, $\Delta_{Q}^{*}=w_{03}\left(L_{0}\right) / \sqrt{2 \gamma}$, in Fig. 6(b), both as functions of the conjugate bond zone size, $a^{*}$. Plots of the corresponding degradation of the global stiffness, $K_{Q}^{*}=Q^{*} / \Delta_{Q}^{*}$, as a function of the conjugate bond zone size are displayed in Fig. 6(c). As for the case of applied in-plane tension, it may be seen that each of the curves is piecewise continuous with discontinuities occurring at the boundary of each particular step within the tapered region. It is also seen that the threshold values of the applied load and corresponding edge deflection are increased as a result of the tapering but drop abruptly at the boundary of each step. It is seen, by comparison of the paths, that when the threshold levels suddenly drop they drop to what would be the extension of the path of a patch of more moderate taper possessing the equivalent stiffness within the tapered region. It is seen, from Fig. 6(a), that while introducing a finite taper at the edge of the patch raises the initial threshold level of the applied load with increasing taper, for a given initial size of the bonded region, once debonding ensues it progresses in an unstable and catastrophic manner for force controlled loading. This characteristic is evidently independent of the presence or degree of tapering. The case of deformation controlled loading, however, offers a more interesting scenario. Upon consideration of Fig. 6(b), it may be seen that, as for the applied force, the threshold level of the transverse edge displacement is raised according to the degree of taper, and that the segments of the curves following the drops at step boundaries continue as equivalent extensions of less severely tapered patches according to the patch stiffness within the tapered region. It is seen that, as for the untapered case, once the threshold level is achieved, debonding ensues and progresses in a catastrophic manner, in an unstable followed by a stable manner, or in a stable manner depending upon the initial size of the bonded region for a deflection controlled test. It may be noted, however, that while introducing tapering is seen to raise the corresponding threshold level for a given initial bond zone size, once debonding is initiated it may progress in an unstable manner to a greater extent than it would have had there been milder tapering or no tapering at all. Thus, the introduction of tapering is seen to destabilize the debonding process for deflection controlled loading for this configuration.

\section{Concluding remarks}

The problem of 1-D edge debonding of layerwise step-tapered patches from base structures of both flat and curved geometries, has been considered. A self-consistent formulation has been presented resulting in mathematical models of the composite structure (both flat and curved) as an assemblage of the primitive structures corresponding to the individual layers of the patch and the base structure modeled as individual shells or plates accordingly, both in the intact and debonded segments of the structure. Also included are the conditions which establish the location of the propagating boundary of the bonded region and the corresponding boundary of an adjacent 
(a)

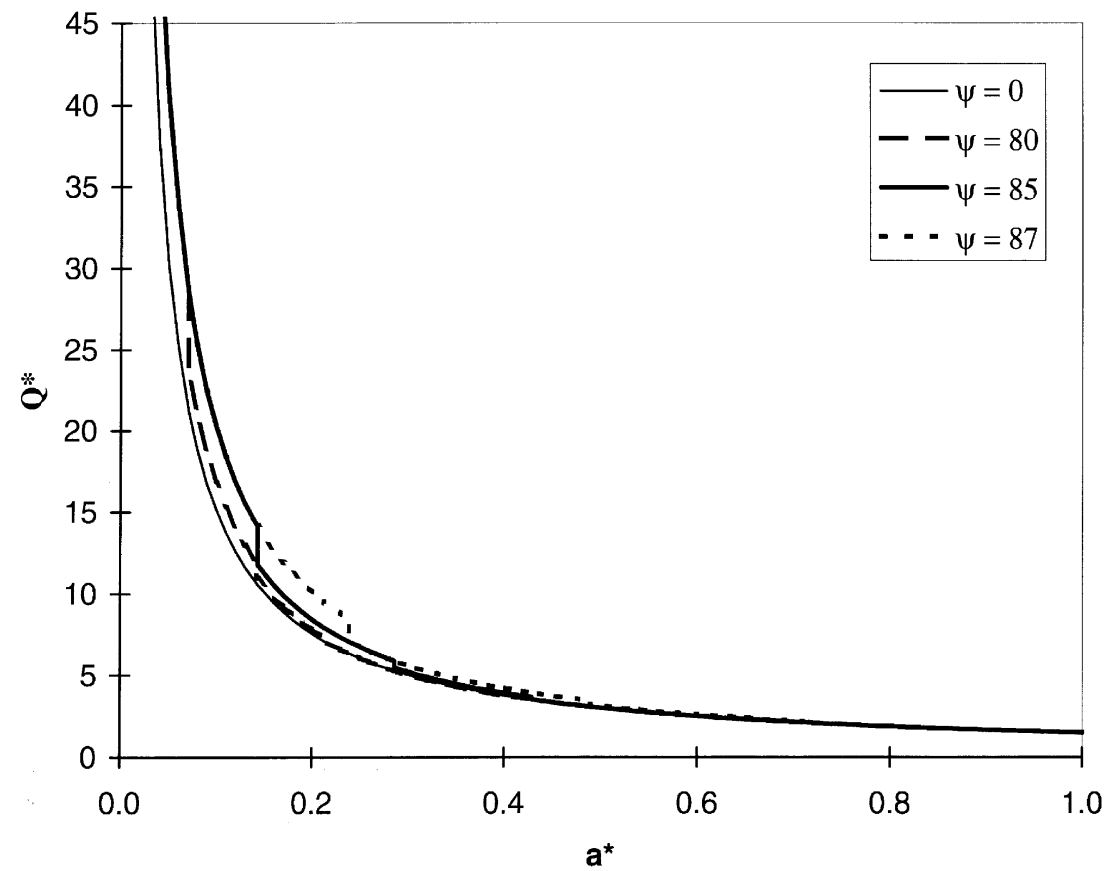

(b)

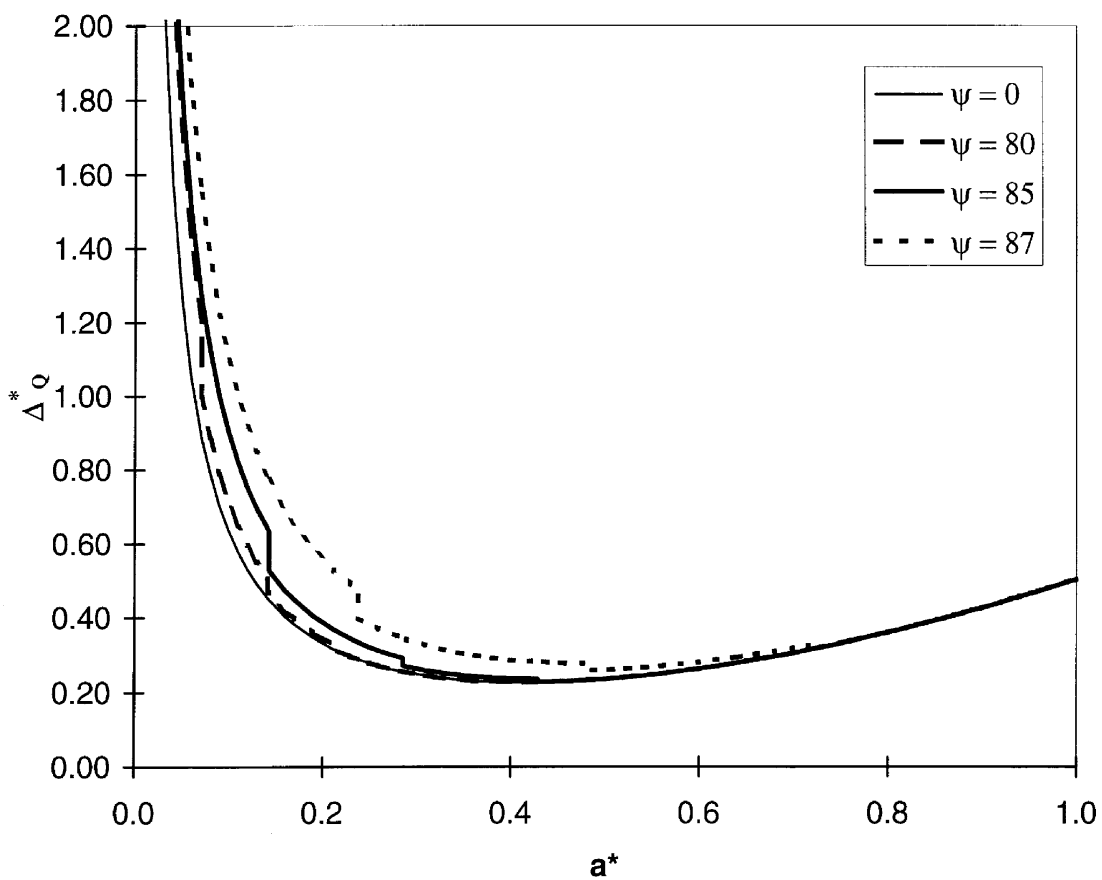

Fig. 6. Threshold curves for plates, with patches of selected taper angles, subjected to three-point loading. (a) Critical load intensity vs conjugate bond zone size, (b) critical transverse edge deflection vs conjugate bond zone size, (c) associated global stiffness as a function conjugate bond zone size. 
(c)

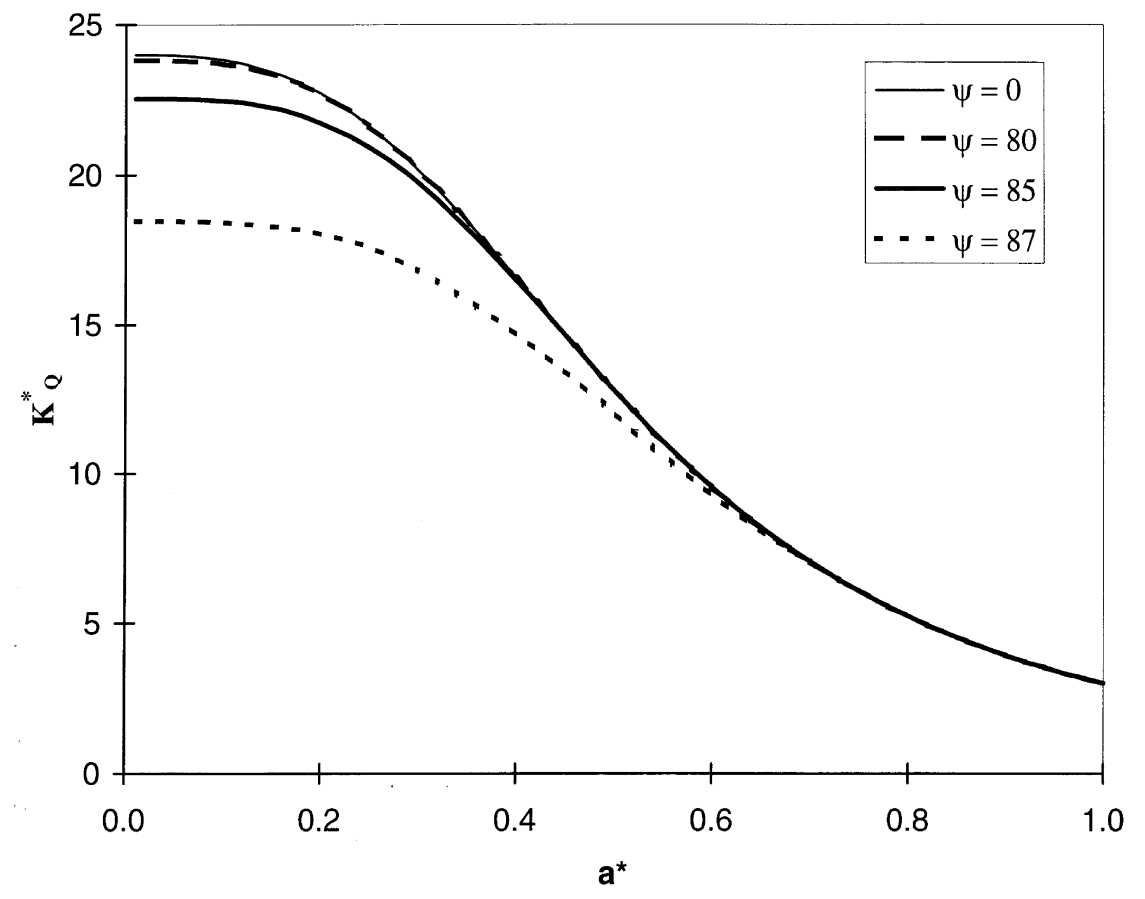

Fig. 6-continued.

region of sliding contact. The former includes the energy release rates expressed in terms of the assembled and primitive structural segments. Three general loading types were considered; (i) applied in-plane/circumferential tension, (ii) three-point transverse loading, and (iii) applied internal/transverse pressure.

It was shown, based on a linearization of the nonlinear formulation presented, that a contact zone can only be present for the case of applied internal/transverse pressure, and that when it occurs it will cover the entire debonded region of the patch. In addition, the case of edge point contact of the debonded segment of the patch was considered.

Results of numerical simulations, based on analytical solutions, were presented for the case of applied in-plane tension and three point transverse loading for selected flat plates, the former for both clamped and pinned supports at the edges of the base plate. It was seen that while tapering of the edge of the patch generally raised the threshold level of the load for the two loading types for which results were presented, in some cases it transformed the debonding process to be unstable and possibly catastrophic in certain circumstances for which debonding was stable without the taper.

It is evident that the effects of tapering must therefore be carefully studied. Toward this end, an extensive analysis and intricate results pertaining to the complex debonding scenarios occurring for pressure loaded structures, both flat and curved, are presented in Part 2 of this study (Karlsson and Bottega, 1998). 


\section{Appendix A : Bond zone stresses}

The expressions for the stresses (Lagrange multipliers) occurring at the interface between the patch and the base structure within the intact segment of the composite structure, $s \in S_{1}$, are found from the (linearized) equations for the primitive structures corresponding to that region (not presented) together with the corresponding equations for the intact composite structure, eqns $(13 \mathrm{a}, \mathrm{b})$ for $i=1$. After some manipulation, we find

$$
\begin{aligned}
& \sigma_{1}^{(1)}(s)=-\alpha_{(q)}^{*} p_{0}+k\left[n_{(q)}^{*} N_{0}+m_{(q)}^{*} \kappa_{1}^{*}(s)\right], \quad(k=0,1) \quad\left(s \in S_{1}\right) \\
& \tau_{1}^{(1)}(s)=m_{(q)}^{*} \kappa_{1}^{\prime}(s),
\end{aligned}
$$

where

$$
\begin{aligned}
& \alpha_{(q)}^{*}=\left\{A_{(q)}^{\circ}-\rho_{(q)}^{*} B_{(q)}^{\circ}\right\} / D_{(q)}^{*}, \\
& n_{(q)}^{*}=\alpha_{(q)}^{*}+\rho_{(q)}^{*}\left\{B_{(q)}^{\circ} / B_{(q)}^{*}\right\}-\left\{C_{(q)}^{\circ} / C_{(q)}^{*}\right\}, \\
& m_{(q)}^{*}=\rho_{(q)}^{*} C_{(q)}^{\circ}-B_{(q)}^{\circ} .
\end{aligned}
$$

\section{Appendix B : Formulation for edge contact}

For the case of edge-point contact, a constraint functional of the form given by

$$
\Lambda_{c}=V_{0}\left[w_{02}\left(L_{1}\right)-w_{12}\left(L_{1}\right)\right]
$$

is added to the functional given by eqn (8), with $\sigma_{1}^{(2)}=\tau_{1}^{(2)}=0$, where the quantity $V_{0}$ is a Lagrange multiplier. For this case, the debonded segments of the base structure and the patch for $s \in S_{2}$ are separated and the exterior lift zone, region $S_{31}$, no longer exists. Correspondingly, the governing differential equations pertaining to these regions take the forms of eqns (14a)-(15b) when subscripted accordingly, while the boundary and matching conditions at $s=0, s=a$ and $s=L_{0}$ given by eqns (22a)-(23g) and (26a)-(26c) retain the same general form, with the parameters corresponding to region 2 with a superscript * interpreted in the present context, and eqns $(17 \mathrm{~b}$ 2) and (17d-2) no longer valid.

The boundary conditions corresponding to the membrane force and bending moment at the patch edge, eqns (25a) and (25b), respectively, are maintained for the present case with the index three replaced by two. The shear balance at the boundary between regions $S_{2}$ and $S_{3}$, however, is adjusted for the present case as a result of the inclusion of the constraint functional defined by eqn (B1). It takes the form

$$
\left[M_{3}^{\prime}-N_{3} w_{3}^{* \prime}\right]_{s=L_{1}}-\left[M_{2}^{\prime}-N_{2} w_{2}^{* \prime}\right]_{s=L_{1}}=\left[M_{2}^{\circ \prime}-N_{2}^{\circ} w_{2}^{\circ \prime}\right]_{s=L_{1}}=V_{0} \geqslant 0,
$$

and is accompanied by the matching condition

$$
w_{2}^{*}\left(L_{1}\right)=w_{2}^{\circ}\left(L_{1}\right),
$$

where the Lagrange multiplier, $V_{0}$, corresponds to the normalized contact force. The inequality incorporated into eqn (B2) is imposed so as to restrict solutions to correspond to the physically 
realizable configurations associated with compressive contact. In addition, for the case of edge contact, the conditions

$$
\begin{aligned}
& u_{2}^{*}\left(L_{1}\right)=\mu_{3}^{*}\left(L_{1}\right), \quad N_{2}\left(L_{1}\right)=N_{3}\left(L_{1}\right), \\
& w_{2}^{*}\left(L_{1}\right)=w_{3}^{*}\left(L_{1}\right), \quad w_{2}^{* \prime}\left(L_{1}\right)=w_{3}^{* \prime}\left(L_{1}\right), \quad M_{2}\left(L_{1}\right)=M_{3}\left(L_{1}\right),
\end{aligned}
$$

replace the conditions given by eqns (24).

Finally, for the case of edge-point contact, the transversality condition at $r=a$ and the associated energy release rate takes the same general form as defined in eqns (29b) but with the index three replaced by two. Incorporation of conditions (32) yields the form

$$
\mathscr{G}_{\mathscr{B}}\{a\} \rightarrow\left[\frac{1}{2} D_{0} \kappa_{02}^{2}+\frac{1}{2} D_{(q)}^{\circ} \kappa_{2}^{\circ 2}-\frac{1}{2} D_{(q)}^{*} \kappa_{1}^{* 2}+\frac{1}{2 C_{(q)}^{(e)}} N_{0}^{2}\right]_{s=a}=2 \gamma
$$

in lieu of eqn $\left(29^{\prime} b\right)$. The integrability condition, for the present case, remains the same as that given by eqn (34).

\section{Acknowledgements}

The authors wish to thank D. W. Oplinger of the Federal Aviation Administration for his support and encouragement. This work was supported by the FAA through the Rutgers University Center for Computational Modeling of Aircraft Structures (CMAS).

\section{References}

Armanios, E.A., Parnas, L., 1989. Delamination analysis of tapered laminated composites under tensile loading. In: O'Brien, T.K. (Ed.). Composite Materials: Fatigue and Fracture, 3rd volume. ASTM STP 1110, Proceedings of the 3rd Symposium on Composite Materials : Fatigue and Fracture, Lake Buenovista, Fla. 6-7 November, pp. 400-438.

Baker, A.A., 1993. Repair efficiency in fatigue-cracked aluminum components reinforced with boron/epoxy patches. Fatigue and Fracture of Engineering Structures 16, 753-765.

Bottega, W.J., 1995. Separation failure in a class of bonded plates. Composite Structures 30 (3), 253-269.

Bottega, W.J., Loia, M.A., 1996. Edge debonding in patched cylindrical panels. International Journal of Solids and Structures 33 (5), 3755-3777.

Bottega, W.J., Loia, M.A., 1997. Axisymmetric edge debonding in patched plates. International Journal of Solids and Structures 34 (18), 2255-2289.

Botting, A.D., Vizzini, A.J., Lee, S.W., 1996. Effect of ply-drop configuration on delamination strength of tapered composite structures. AIAA Journal 40 (8), 1650-1656.

Curry, J.M., Johnson, E.R., Starnes, J.H., 1992. Effect of dropped plies on the strength of graphite-epoxy laminates. AIAA Journal 30 (2), 449-456.

Fish, J.C., Lee, S.W., 1989. Delamination of tapered composite structures. Engineering Fracture Mechanics 40 (1), $43-$ 54.

Karlsson, A.M., Bottega, W.J., 1998. On the detachment of step-tapered doublers: Part 2 - evolution of pressure loaded structures. International Journal of Solids and Structures 36 (11), 1625-1651.

Loia, M.A., Bottega, W.J., 1995. On planar-tensile representation of edge debonding in patched panels under pressure. International Journal of Adhesion and Adhesives 15 (4), 211-217.

Raizenne, M.D., Heath, J.B.R., Gaudertt, P.C., 1995. Failure analysis of bonded boron doublers. Proceedings of the 
National Research Council of Canada Symposium on Composite Repair of Aircraft Structures, University of British Columbia, Vancouver, 9-10 August, pp. 10.1-10.30.

Roderick, G.L., 1980. Prediction of cyclic growth of cracks and debonds on aluminum sheets reinforced with boron/ epoxy. In : Lenoe, E.M., Oplinger, D.W., Burke, J.J. (Ed.). Fibrous Composites in Structural Design. Plenum Press, New York, pp. 467-481.

Salpekar, S.A., Rau, I.S., O’Brien, T.K., 1991. Strain energy release rate analysis of delamination in a tapered laminate subjected to tensile load. Journal of Composite Materials 25, 118-143.

Sih, G.C., Hong, T.B., 1989. Integrity of edge-debonded patch on cracked panel. Theoretical and Applied Fracture Mechanics 12, 121-143. 